\title{
STABILITY OF THE GAUGE EQUIVALENT CLASSES IN STATIONARY INVERSE TRANSPORT
}

\author{
STEPHEN MCDOWALL, PLAMEN STEFANOV, AND ALEXANDRU TAMASAN
}

\begin{abstract}
For anisotropic attenuating media, the albedo operator determines the scattering and the attenuating coefficients up to a gauge transformation. We show that such a determination is stable.
\end{abstract}

\section{INTRODUCTION}

This paper concerns the problem of recovering the absorption and scattering properties of a bounded, convex medium $\Omega \subset \mathbb{R}^{n}, n \geq 2$ from the spatial-angular measurements of the density of particles at the boundary $\partial \Omega$. Provided that the particles interact with the medium but not with each other, the radiation transfer in the steady-state can be modeled by the transport equation

$$
-\theta \cdot \nabla u(x, \theta)-a(x, \theta) u(x, \theta)+\int_{S^{n-1}} k\left(x, \theta^{\prime}, \theta\right) u\left(x, \theta^{\prime}\right) d \theta^{\prime}=0,
$$

for $x \in \Omega$ and $\theta \in S^{n-1}$; see, e.g. [10, 25. The function $u(x, \theta)$ represents the density of particles at $x$ traveling in the direction $\theta, a(x, \theta)$ is the attenuation coefficient at $x$ for particles moving in the direction of $\theta$, and $k\left(x, \theta^{\prime}, \theta\right)$ is the scattering coefficient (or the collision kernel) which accounts for particles from an arbitrary direction $\theta^{\prime}$ which scatter in the direction of travel $\theta$. Let $\Gamma_{ \pm}$denote the incoming and outgoing "boundary"

$$
\Gamma_{ \pm}:=\left\{(x, \theta) \in \partial \Omega \times S^{n-1}: \quad \pm \theta \cdot n(x)>0\right\},
$$

$n(x)$ being the outer unit normal at a boundary point $x \in \partial \Omega$. The medium is probed with the given radiation

$$
\left.u\right|_{\Gamma_{-}}=f_{-} .
$$

The exiting radiation $\left.u\right|_{\Gamma_{+}}$is detected thus defining the albedo operator $\mathcal{A}$ that takes the incoming flux $f_{-}$to the outgoing flux $\left.u\right|_{\Gamma_{+}}$, i.e. $\mathcal{A}\left[f_{-}\right]:=\left.u\right|_{\Gamma_{+}}$.

In general, the boundary value problem (11) and (3) may not be uniquely solvable but it has a unique solution under some physically relevant subcritical conditions like (35), (36), or (81). We note, however, that for sufficiently regular coefficients, the problem has unique solution for generic $(a, k)$, see [30, 28].

One of the inverse boundary value problems in transport theory is to recover the attenuation coefficient $a$ and the scattering kernel $k$ from knowledge of the albedo

AMS Subject Classification: 35R30,78A46.

First author partly supported by NSF Grant No. 0553223.

Second author partly supported by NSF Grant No. 0554065. 
operator $\mathcal{A}$. This problem has been solved under some restrictive assumptions (e.g. $k$ of a special type or independent of a variable) in [1, 2, 3, 15, 18, 19, 20, 31, 32. In three or higher dimensions, uniqueness and reconstruction results for general $k$ and $a=a(x)$ were established in [12]. The general approach is based on the study of the singularities of the fundamental solution of (11) (see also [9]), and the singularities of the Schwartz kernel of $\mathcal{A}$. Stability estimates for $k$ of special type were established in [27, 33]; and recently, for general $k$, in [7]. Uniqueness and reconstruction results in a Riemannian geometry setting, including recovery of a simple metric, were established in 21. Similar results for the time-dependent model were established in [11, and in [15] for the Riemannian case. In planar domains the work in [29] shows stable determination of the isotropic absorption and small scattering, and an extension to simple Riemannian geometry is given in [22. Also in two dimensional domains we point out that the recovery of $k$ is only known under smallness conditions which are more restrictive than what is needed to solve the direct problem; e.g. more restrictive than (35) or (36) below. On the other hand, in the time-dependent case, the extra variable allows us to treat the planar case without such restrictions, see [11]. We also mention here the recent works [8, 16, 17], in which the coefficients are recovered from angularly averaged measurements rather than from the knowledge of the whole albedo operator $\mathcal{A}$. For an exhaustive account on the inverse transport problem we refer to the review paper [6].

The above mentioned results concern media with directionally independent absorption $a=a(x)$, except for transport with variable speed when the attenuation may depend on $|v|, a=a(x,|v|)$.

The attenuation accounts not only for the absorption of particles, but also for the loss of particles due to the scattering. In the physical case in which $k$ depends on two independent directions, the attenuation is inherently anisotropic $a=a(x, \theta)$. However, in an anisotropic attenuating media, the unique determination of the coefficients from boundary measurements no longer holds: In [28] it is shown that the albedo operator determines the pairs of coefficients up to a gauge transformation; see (4) below. This non-uniqueness motivates the following definition.

Definition 1.1. Two pairs of coefficients $(a, k)$ and $(\tilde{a}, \tilde{k})$ are called gauge equivalent if there exists a positive $\phi \in L^{\infty}\left(\Omega \times S^{n-1}\right)$ with $\theta \cdot \nabla_{x} \phi(x, \theta) \in L^{\infty}\left(\Omega \times S^{n-1}\right)$ and $\phi=1$ for $x \in \partial \Omega$ such that

$$
\tilde{a}(x, \theta)=a(x, \theta)-\theta \cdot \nabla_{x} \log \phi(x, \theta), \quad \tilde{k}\left(x, \theta^{\prime}, \theta\right)=\frac{\phi(x, \theta)}{\phi\left(x, \theta^{\prime}\right)} k\left(x, \theta^{\prime}, \theta\right) .
$$

We denote the equivalence class of $(a, k)$ by $\langle a, k\rangle$, and the equivalence relation itself by $\sim$.

The relation defined above is reflexive since $(a, k) \sim(a, k)$ via $\phi \equiv 1$; it is symmetric since $(a, k) \sim(\tilde{a}, \tilde{k})$ via $\phi$ yields $(\tilde{a}, \tilde{k}) \sim(a, k)$ via $1 / \phi$; and it is transitive since if $(a, k) \sim(\tilde{a}, \tilde{k})$ via $\phi$ and $(\tilde{a}, \tilde{k}) \sim\left(a^{\prime}, k^{\prime}\right)$ via $\tilde{\phi}$ then $(a, k) \sim\left(a^{\prime}, k^{\prime}\right)$ via $\phi \tilde{\phi}$.

The main result in [28] is that, in dimensions $n \geq 3, \mathcal{A}=\tilde{\mathcal{A}}$ if and only if $(a, k) \sim$ $(\tilde{a}, \tilde{k})$, i.e., uniqueness up to gauge transformations. The uniqueness up to the gauge transformation extends naturally to refractive media and to dimension two, see [23]. 
In this paper we study the question of stability of the determination of the gauge equivalent classes. Let $\left(M,\|\cdot\|_{M}\right)$ and $\left(N,\|\cdot\|_{N}\right)$ be Banach spaces in which the attenuation and, respectively, the scattering kernel are considered, $(a, k),(\tilde{a}, \tilde{k}) \in$ $M \times N$. The distance $\Delta$ between equivalence classes with respect to $M \times N$ is given by the infimum of the distances between all possible pairs of representatives. More precisely,

$$
\Delta(\langle a, k\rangle,\langle\tilde{a}, \tilde{k}\rangle):=\inf _{\left(a^{\prime}, k^{\prime}\right) \in\langle a, k\rangle,\left(\tilde{a}^{\prime}, \tilde{k}^{\prime}\right) \in\langle\tilde{a}, \tilde{k}\rangle} \max \left\{\left\|a^{\prime}-\tilde{a}^{\prime}\right\|_{M},\left\|k^{\prime}-\tilde{k}^{\prime}\right\|_{N}\right\} .
$$

For $n \geq 3$ we work within the class of coefficients

$$
(a, k) \in L^{\infty}\left(\Omega \times S^{n-1}\right) \times L^{\infty}\left(\Omega \times S^{n-1} ; L^{1}\left(S^{n-1}\right)\right) .
$$

For two dimensional domains $(n=2)$ both coefficients are assumed bounded:

$$
(a, k) \in L^{\infty}\left(\Omega \times S^{1}\right) \times L^{\infty}\left(\Omega \times S^{1} \times S^{1}\right) .
$$

The following norms are used throughout

$$
\begin{aligned}
\|a\|_{\infty} & =\operatorname{ess} \sup _{(x, \theta) \in \Omega \times S^{n-1}}|a(x, \theta)| \\
\|k\|_{\infty, 1} & =\operatorname{ess} \sup _{\left(x, \theta^{\prime}\right) \in \Omega \times S^{n-1}} \int_{S^{n-1}}\left|k\left(x, \theta^{\prime}, \theta\right)\right| d \theta, \\
\|k\|_{\infty} & =\operatorname{ess} \sup _{\left(x, \theta^{\prime}, \theta\right) \in \Omega \times S^{1} \times S^{1}}\left|k\left(x, \theta^{\prime}, \theta\right)\right| \\
\|k\|_{1} & =\int_{\Omega} \int_{S^{n-1}} \int_{S^{n-1}}\left|k\left(x, \theta^{\prime}, \theta\right)\right| d x d \theta^{\prime} d \theta .
\end{aligned}
$$

Note that the gauge transformations preserve the class of coefficients in (6) or (7).

In Section 2 we reduce the original inverse problem in $\Omega$ to the inverse problem of transport in a larger (strictly convex) domain $B_{R} \supset \bar{\Omega}$, where the attenuation and scattering coefficients are extended by zero in $B_{R} \backslash \Omega$. More precisely we show that the difference of two albedo operators realizes an isometry when transported from $\partial \Omega$ to $\partial B_{R}$. For simplicity, the larger domain is a ball but this is not essential. Let $(\tilde{a}, \tilde{k})$ be another pair of admissible coefficients for which the forward problem in $\Omega$ is well posed and let $\tilde{\mathcal{A}}$ denote the corresponding albedo operators. Set $a=\tilde{a}=0$ and $k=\tilde{k}=0$ in $B_{R} \backslash \bar{\Omega}$. Then the forward problems in $B_{R}$ are also well posed and let $\mathcal{A}^{R}$ and $\tilde{\mathcal{A}}^{R}$, denote the corresponding albedo operators respectively. The boundary data is considered on

$$
\Gamma_{ \pm}^{R}:=\left\{(x, \theta) \in \partial B_{R} \times S^{n-1}: \quad \pm \theta \cdot n(x)>0\right\},
$$

$n(x)$ now being the outer unit normal at a boundary point $x \in \partial B_{R}$. Provided that the forward problem is well-posed in $L^{p}, 1 \leq p \leq \infty$, we show that

$$
\|\mathcal{A}-\tilde{\mathcal{A}}\|_{\mathcal{L}\left(L^{p}\left(\Gamma_{-} ; d \xi\right) ; L^{p}\left(\Gamma_{+} ; d \xi\right)\right)}=\left\|\mathcal{A}^{R}-\tilde{\mathcal{A}}^{R}\right\|_{\mathcal{L}\left(L^{p}\left(\Gamma_{-}^{R} ; d \xi^{R}\right) ; L^{p}\left(\Gamma_{+}^{R} ; d \xi R\right)\right)} .
$$

In (9) we used $d \xi=|n(x) \cdot \theta| d \mu(x) d \theta$, where $d \theta$ is the normalized measure on the sphere, $d \mu(x)$ is the induced Lebesgue measure on $\partial \Omega$ and $n(x)$ is the unit outer normal at some $x \in \partial \Omega$. Similarly, $d \xi^{R}=|n(x) \cdot \theta| d \mu^{R}(x) d \theta$, where $d \mu^{R}(x)$ is the induced Lebesgue measure on $\partial B_{R}$. 
Consequently, we may consider the data (albedo operators) given directly on the $\partial B_{R}$ and drop $R$ from their notation. The isometry (9) with $p=1$ is used for domains in three or higher dimensions. For brevity let

$$
\|\mathcal{A}-\tilde{\mathcal{A}}\|:=\|\mathcal{A}-\tilde{\mathcal{A}}\|_{\mathcal{L}\left(L^{1}\left(\Gamma_{-} ; d \xi\right) ; L^{1}\left(\Gamma_{+} ; d \xi\right)\right)} .
$$

Let $\tau_{ \pm}(x, \theta)$ be the travel time it takes a particle at $x \in B_{R}$ to reach the boundary $\partial B_{R}$ while moving in the direction of $\pm \theta$ and define $\tau(x, \theta)=\tau_{-}(x, \theta)+\tau_{+}(x, \theta)$. Since we work with unit-speed velocities, $\tau(x, \theta) \leq 2 R$. Moreover, since $\operatorname{dist}\left(\bar{\Omega}, \partial B_{R}\right)>0$, we have

$$
c_{R}:=\inf \left\{\tau(x, \theta):(x, \theta) \in \bar{\Omega} \times S^{n-1}\right\}>0 .
$$

Note that we could make $c_{R}=1$ at the expense of a sufficiently large radius $R$.

For domains in three or higher dimensions, and for $\Sigma, \rho>0$ we consider the class

$$
U_{\Sigma, \rho}:=\left\{(a, k) \text { as in (7) : }\|a\|_{\infty} \leq \Sigma,\|k\|_{\infty, 1} \leq \rho\right\} .
$$

The main result of stability of gauge equivalent classes is the following.

Theorem 1.2. Let $(a, k),(\tilde{a}, \tilde{k}) \in U_{\Sigma, \rho}$ be such that the corresponding forward problems are well posed. Then

$$
\Delta(\langle a, k\rangle,\langle\tilde{a}, \tilde{k}\rangle) \leq C\|\mathcal{A}-\tilde{\mathcal{A}}\|,
$$

where $\Delta$ is with respect to $L^{\infty} \times L^{1}$, and $C$ is a constant depending only on $\Sigma, \rho, c_{R}$ and $R$. More precisely, there exists a representative $\left(a^{\prime}, k^{\prime}\right) \in\langle a, k\rangle$ such that

$$
\begin{aligned}
\left\|a^{\prime}-\tilde{a}\right\|_{\infty} & \leq C\|\mathcal{A}-\tilde{\mathcal{A}}\|, \\
\left\|k^{\prime}-\tilde{k}\right\|_{1} & \leq C\|\mathcal{A}-\tilde{\mathcal{A}}\|,
\end{aligned}
$$

where

$$
C=\max \left\{\pi R e^{2 R \Sigma}\left(1+2 \rho e^{4 R \Sigma}\right), e^{4 R \Sigma} / c_{R}\right\} .
$$

For the stability of the equivalence classes in two dimensional domains we need a more refined notion of distance between the albedo operators: Following [29, Proposition 1], the Schwartz kernel of a albedo operator $\mathcal{A}$ admits the singular decomposition:

$$
\alpha=\frac{A\left(x^{\prime}, \theta^{\prime}\right)}{n(x) \cdot \theta} \delta_{\left\{x^{\prime}+\tau_{+}\left(x^{\prime}, \theta^{\prime}\right) \theta^{\prime}\right\}}(x) \delta_{\left\{\theta^{\prime}\right\}}(\theta)+\beta\left(x, \theta, x^{\prime}, \theta^{\prime}\right),
$$

where

$$
A\left(x^{\prime}, \theta^{\prime}\right)=\exp \left(-\int_{0}^{\tau_{+}\left(x^{\prime}, \theta^{\prime}\right)} a\left(x^{\prime}+t \theta^{\prime}, \theta^{\prime}\right) d t\right)
$$

and $\left|\theta \times \theta^{\prime}\right| \beta \in L^{\infty}\left(\Gamma_{+}^{R} \times \Gamma_{-}^{R}\right)$; see also Section 6 .

Let $\tilde{A}, \tilde{\beta}$ be the coefficients corresponding to the decomposition (16) of another albedo operator $\tilde{\mathcal{A}}$. We define

$$
\|\mathcal{A}\|_{*}=\max \left\{\|A\|_{\infty} ;\left\|\left|\theta \times \theta^{\prime}\right| \beta\right\|_{\infty}\right\} .
$$


In Section 2 we show that the isometric transportation from $\partial \Omega$ to $\partial B_{R}$ holds in the new operator norm, i.e.,

$$
\|\mathcal{A}-\tilde{\mathcal{A}}\|_{*}=\left\|\mathcal{A}^{R}-\tilde{\mathcal{A}}^{R}\right\|_{*}
$$

In the two dimensional case, for $\Sigma, \rho>0$, we consider the smaller class

$$
V_{\Sigma, \rho}:=\left\{(a, k) \text { as in (7) : }\|a\|_{\infty} \leq \Sigma,\|k\|_{\infty} \leq \rho\right\} .
$$

Theorem 1.3. For any $\Sigma>0$, there exists $0<\rho \leq 1$ depending only on $\Sigma, R$ and $c_{R}$, such that the following holds: If $(a, k),(\tilde{a}, \tilde{k}) \in V_{\Sigma, \rho}$, then

$$
\Delta(\langle a, k\rangle,\langle\tilde{a}, \tilde{k}\rangle) \leq C\|\mathcal{A}-\tilde{\mathcal{A}}\|_{*},
$$

where $\Delta$ is with respect to $L^{\infty} \times L^{\infty}$, and $C$ is a constant depending only on $\Sigma, \rho, c_{R}$ and $R$.

The proofs of Theorem 1.2 and 1.3 are based on the analysis of the singularities of the Schwartz kernel of $\mathcal{A}$ as in [12] and, respectively, [29]. We also use an extended version of an estimate in [7], see Section 4 below.

One can formulate and prove similar results in the case where the velocity belongs to an open subspace of $\mathbb{R}^{n}$, i.e., the speed can change, as in [7, 12]. We restrict ourselves to the fixed speed case $(|\theta|=1)$ for the sake of simplicity of the exposition.

\section{IsOMETRIC TRANSPORTATION OF THE ALBEDO OPERATORS FROM $\partial \Omega$ TO $\partial B_{R}$}

Recall that $\Omega$ is strictly convex with $\bar{\Omega} \subset B_{R}$, and $\mathcal{A}$ is the albedo operator for the radiation transport in $\Omega$. The coefficients $(a, k)$ are extended by zero in $B_{R} \backslash \bar{\Omega}$ and let $\mathcal{A}_{R}$ be the albedo operator corresponding to the radiative transport in $B_{R} \times S^{n-1}$, which takes functions on $\Gamma_{-}^{R}$ to functions on $\Gamma_{+}^{R}$; see (8) . Recall that $\tau_{ \pm}(x, \theta)$ is the travel time it takes a particle at $x \in B_{R}$ to reach the boundary $\partial B_{R}$ while moving in the direction of $\pm \theta$.

We consider next the set of "projections" of $\Gamma_{ \pm}$onto $\Gamma_{ \pm}^{R}$ defined by

$$
\tilde{\Gamma}_{ \pm}^{R}:=\left\{\left(x \pm \tau_{ \pm}(x, \theta) \theta, \theta:(x, \theta) \in \Gamma_{ \pm}\right\} \subsetneq \Gamma_{ \pm}^{R}\right.
$$

and the transportation maps

$$
\begin{aligned}
& {[\mathcal{T} f]\left(x^{\prime}, \theta^{\prime}\right):=f\left(x^{\prime}-\tau_{-}\left(x^{\prime}, \theta^{\prime}\right) \theta^{\prime}, \theta^{\prime}\right), \quad \forall\left(x^{\prime}, \theta^{\prime}\right) \in \Gamma_{-}} \\
& {[\tilde{\mathcal{T}} f]\left(x+\tau_{+}(x, \theta) \theta, \theta\right):=f(x, \theta), \quad \forall(x, \theta) \in \Gamma_{+} .}
\end{aligned}
$$

$\mathcal{T}$ takes maps defined on $\tilde{\Gamma}_{-}^{R}$ to maps on $\Gamma_{-}$, whereas $\tilde{\mathcal{T}}$ takes maps defined on $\Gamma_{+}$to maps on $\tilde{\Gamma}_{+}^{R}$.

Proposition 2.1. For any $1 \leq p \leq \infty$, the maps

$$
\begin{aligned}
& \mathcal{T}: L^{p}\left(\tilde{\Gamma}_{-}^{R} ; d \xi\right) \rightarrow L^{p}\left(\Gamma_{-} ; d \xi\right), \\
& \tilde{\mathcal{T}}: L^{p}\left(\Gamma_{+} ; d \xi\right) \rightarrow L^{p}\left(\tilde{\Gamma}_{+}^{R} ; d \xi\right)
\end{aligned}
$$

are isomorphisms. 
Proof. From the definitions (23) and (24) we have

$$
\operatorname{ess} \sup _{\tilde{\Gamma}_{-}^{R}} f=\operatorname{ess} \sup _{\Gamma_{-}}[\mathcal{T} f] \text { and } \operatorname{ess} \sup _{\tilde{\Gamma}_{-}^{R}}[\tilde{\mathcal{T}} f]=\operatorname{ess} \sup _{\Gamma_{-}} f .
$$

This proves the isometry for $p=\infty$. For $1 \leq p<\infty$ we have the identities

$$
\begin{array}{r}
\int_{\tilde{\Gamma}_{-}^{R}}|f(x, \theta)|^{p} d \tilde{\xi}(x, \theta)=\int_{\Gamma_{-}}\left|[\mathcal{T} f]\left(x^{\prime}, \theta\right)\right|^{p} d \xi\left(x^{\prime}, \theta\right), \\
\int_{\tilde{\Gamma}_{+}^{R}}|\tilde{\mathcal{T}} f(x, \theta)|^{p} d \tilde{\xi}(x, \theta)=\int_{\Gamma_{+}}\left|f\left(x^{\prime}, \theta\right)\right|^{p} d \xi\left(x^{\prime}, \theta\right)
\end{array}
$$

obtained by the change of variables $x=x^{\prime}-\tau_{-}\left(x^{\prime}, \theta\right)$ and $x=x^{\prime}+\tau_{+}\left(x^{\prime}, \theta\right)$ respectively.

Proposition 2.2. Let $(a, k)$ be an admissible pair for the transport in $\Omega$ such that the forward problem is well-posed in $L^{p}\left(\Omega \times S^{n-1}\right), 1 \leq p \leq \infty, n \geq 2$, and let $\mathcal{A}$ be the corresponding albedo operator. Extend the coefficients by zero in $B_{R} \backslash \bar{\Omega}$. Then the forward problem in $L^{p}\left(B_{R} \times S^{n-1}\right), 1 \leq p \leq \infty$, is also well posed and let $\mathcal{A}^{R}$ be the corresponding albedo operator. For any $f \in L^{p}\left(\Gamma_{-}^{R}\right)$ we have

$$
\mathcal{A}^{R}[f](x, \theta)= \begin{cases}f\left(x-\tau_{-}(x, \theta) \theta, \theta\right), & \text { if }(x, \theta) \in \Gamma_{+}^{R} \backslash \tilde{\Gamma}_{+}^{R}, \\ \tilde{\mathcal{T}} \mathcal{A} \mathcal{T} f(x, \theta), & \text { if }(x, \theta) \in \tilde{\Gamma}_{+}^{R} .\end{cases}
$$

Proof. The relation (30) is a direct consequence of the fact that in $B_{R} \backslash \bar{\Omega}$, where the coefficients vanish, the solution $u(x, \theta)$ of the transport equation is constant in $x$ along the lines in the direction of $\theta$.

Proposition 2.3. Let $(a, k)$ and $(\tilde{a}, \tilde{k})$ be admissible pairs for the transport in $\Omega$ such that the forward problem is well-posed in $L^{p}\left(\Omega \times S^{n-1}\right), n \geq 2,1 \leq p \leq \infty$, and let $\mathcal{A}$ and $\tilde{\mathcal{A}}$ be the corresponding albedo operators. Extend the coefficients by zero in $B_{R} \backslash \bar{\Omega}$ and let $\mathcal{A}^{R}$, respectively $\tilde{\mathcal{A}}^{R}$, be their corresponding albedo operators for the transport through $B_{R}$. Then (9) and (19) hold.

Proof. Following (30)

$$
\left(\mathcal{A}^{R}-\tilde{\mathcal{A}}^{R}\right)[f](x, \theta)= \begin{cases}0, & \text { if }(x, \theta) \in \Gamma_{+}^{R} \backslash \tilde{\Gamma}_{+}^{R}, \\ \tilde{\mathcal{T}}[\mathcal{A}-\tilde{\mathcal{A}}] \mathcal{T} f(x, \theta), & \text { if }(x, \theta) \in \tilde{\Gamma}_{+}^{R} .\end{cases}
$$

For $f \in L^{p}\left(\Gamma_{-}^{R}\right)$,

$$
\begin{aligned}
\left\|\left(\mathcal{A}^{R}-\tilde{\mathcal{A}}^{R}\right) f\right\|_{L^{p}\left(\Gamma_{+}^{R}\right)} & =\|\tilde{\mathcal{T}}(\mathcal{A}-\tilde{\mathcal{A}}) \mathcal{T} f\|_{L^{p}\left(\tilde{\Gamma}_{+}^{R}\right)}=\|(\mathcal{A}-\tilde{\mathcal{A}}) \mathcal{T} f\|_{L^{p}\left(\Gamma_{+}\right)} \\
& \leq\|\mathcal{A}-\tilde{\mathcal{A}}\| \cdot\|\mathcal{T} f\|_{L^{p}\left(\Gamma_{-}\right)}=\|\mathcal{A}-\tilde{\mathcal{A}}\| \cdot\|f\|_{L^{p}\left(\tilde{\Gamma}_{-}^{R}\right)} \\
& \leq\|\mathcal{A}-\tilde{\mathcal{A}}\| \cdot\|f\|_{L^{p}\left(\Gamma_{-}^{R}\right)} .
\end{aligned}
$$

Hence $\left\|\mathcal{A}^{R}-\tilde{\mathcal{A}}^{R}\right\| \leq\|\mathcal{A}-\tilde{\mathcal{A}}\|$. 
To prove the converse inequality, let $f_{0}$ be the projection of $f$ on $\tilde{\Gamma}_{+}^{R}$, i.e.

$$
f_{0}:=\left\{\begin{array}{l}
0, \text { on } \Gamma_{+}^{R} \backslash \tilde{\Gamma}_{+}^{R}, \\
f, \text { on } \tilde{\Gamma}_{+}^{R} .
\end{array}\right.
$$

Then

$$
\begin{aligned}
\|(\mathcal{A}-\tilde{\mathcal{A}}) \mathcal{T} f\|_{L^{p}\left(\Gamma_{+}\right)} & =\|\tilde{\mathcal{T}}(\mathcal{A}-\tilde{\mathcal{A}}) \mathcal{T} f\|_{L^{p}\left(\tilde{\Gamma}_{+}^{R}\right)}=\left\|\left(\mathcal{A}^{R}-\tilde{\mathcal{A}}^{R}\right) f\right\|_{L^{p}\left(\Gamma_{+}^{R}\right)} \\
& =\left\|\left(\mathcal{A}^{R}-\tilde{\mathcal{A}}^{R}\right) f_{0}\right\|_{L^{p}\left(\Gamma_{+}^{R}\right)} \leq\left\|\mathcal{A}^{R}-\tilde{\mathcal{A}}^{R}\right\| \cdot\left\|f_{0}\right\|_{L^{p}\left(\Gamma_{-}^{R}\right)} \\
& =\left\|\mathcal{A}^{R}-\tilde{\mathcal{A}}^{R}\right\| \cdot\left\|\left.f\right|_{\tilde{\Gamma}_{-}^{R}}\right\|_{L^{p}\left(\tilde{\Gamma}_{-}^{R}\right)}=\left\|\mathcal{A}^{R}-\tilde{\mathcal{A}}^{R}\right\| \cdot\|\mathcal{T} f\|_{L^{p}\left(\Gamma_{-}\right)} .
\end{aligned}
$$

Since $\mathcal{T}$ is onto $L^{p}\left(\Gamma_{-}\right)$, the inequality above yields $\|\mathcal{A}-\tilde{\mathcal{A}}\| \leq\left\|\mathcal{A}^{R}-\tilde{\mathcal{A}}^{R}\right\|$.

Next, we prove the isometry in the $\|\cdot\|_{*}$-norm. Let $\alpha, \tilde{\alpha}, \alpha^{R}, \tilde{\alpha}^{R}$ be the Schwartz kernels associated with the albedo operators $\mathcal{A}, \tilde{\mathcal{A}}, \mathcal{A}^{R}, \tilde{\mathcal{A}}^{R}$, and let $A, \tilde{A}, A^{R}, \tilde{A}^{R}$ and $\beta, \tilde{\beta}, \beta^{R}, \tilde{\beta}^{R}$ be the coefficients from the corresponding singular decomposition as in (16).

On the one hand, for $f \in C^{\infty}\left(\Gamma_{-}^{R}\right)$ arbitrary, and $(x, \theta) \in \Gamma_{+}$we have:

$$
\begin{aligned}
{[\tilde{\mathcal{T}}(\tilde{\mathcal{A}}-\mathcal{A}) \mathcal{T} f] } & \left(x+\tau_{+}(x, \theta) \theta, \theta\right)=[(\tilde{\mathcal{A}}-\mathcal{A}) \mathcal{T} f](x, \theta) \\
= & \int_{\Gamma_{-}}[\tilde{\alpha}-\alpha]\left(x, \theta, x^{\prime}, \theta^{\prime}\right) \mathcal{T} f\left(x^{\prime}, \theta^{\prime}\right) d \xi\left(x^{\prime}, \theta^{\prime}\right) \\
= & \int_{\Gamma_{-}}[\tilde{\alpha}-\alpha]\left(x, \theta, x^{\prime}, \theta^{\prime}\right) f\left(x^{\prime}-\tau_{-}\left(x^{\prime}, \theta^{\prime}\right), \theta^{\prime}\right) d \xi\left(x^{\prime}, \theta^{\prime}\right) \\
& =\int_{\tilde{\Gamma}_{-}^{R}}[\tilde{\gamma}-\gamma]\left(x, \theta, x_{R}^{\prime}, \theta^{\prime}\right) f\left(x_{R}^{\prime}, \theta^{\prime}\right) d^{R} \xi\left(x_{R}^{\prime}, \theta^{\prime}\right) .
\end{aligned}
$$

In the last equality above we change variables $x_{R}^{\prime}=x^{\prime}-\tau_{-}\left(x^{\prime}, \theta^{\prime}\right) \theta^{\prime}$, and denote the distribution $[\tilde{\gamma}-\gamma]\left(x, \theta, x^{\prime}-\tau_{-}\left(x^{\prime}, \theta^{\prime}\right) \theta^{\prime}, \theta^{\prime}\right):=[\tilde{\alpha}-\alpha]\left(x, \theta, x^{\prime}, \theta^{\prime}\right)$.

On the other hand, from (31), we get

$$
\begin{aligned}
{[\tilde{\mathcal{T}}(\tilde{\mathcal{A}}-\mathcal{A}) \mathcal{T} f] } & \left(x+\tau_{+}(x, \theta) \theta, \theta\right)=\left[\tilde{\mathcal{A}}^{R}-\mathcal{A}^{R}\right] f\left(x+\tau_{+}(x, \theta) \theta, \theta\right) \\
= & \int_{\Gamma_{-}^{R}}\left[\tilde{\alpha}^{R}-\alpha^{R}\right]\left(x+\tau_{+}(x, \theta) \theta, \theta, x_{R}^{\prime}, \theta^{\prime}\right) f\left(x_{R}^{\prime}, \theta^{\prime}\right) d \xi^{R}\left(x^{R}, \theta^{\prime}\right) \\
= & \int_{\tilde{\Gamma}_{-}^{R}}\left[\tilde{\alpha}^{R}-\alpha^{R}\right]\left(x+\tau_{+}(x, \theta) \theta, \theta, x_{R}^{\prime}, \theta^{\prime}\right) f\left(x_{R}^{\prime}, \theta^{\prime}\right) d \xi^{R}\left(x^{R}, \theta^{\prime}\right) .
\end{aligned}
$$

Therefore, in the sense of distributions

$$
\left[\tilde{\alpha}^{R}-\alpha^{R}\right]\left(x+\tau_{+}(x, \theta) \theta, \theta, x^{\prime}-\tau_{-}\left(x^{\prime}, \theta^{\prime}\right) \theta^{\prime}, \theta^{\prime}\right)=[\tilde{\alpha}-\alpha]\left(x, \theta, x^{\prime}, \theta^{\prime}\right)
$$

Independently of the equality (32) above, due to the fact that $a=\tilde{a}=0$ in the $B_{R} \backslash \Omega$, by direct verification in the formula (17), we get

$$
\left[\tilde{A}^{R}-A^{R}\right]\left(x^{\prime}-\tau_{-}\left(x^{\prime}, \theta^{\prime}\right) \theta^{\prime}, \theta^{\prime}\right)=[\tilde{A}-A]\left(x^{\prime}, \theta^{\prime}\right), \quad\left(x^{\prime}, \theta^{\prime}\right) \in \Gamma_{-} .
$$


Now (32) and (33) applied to (16) yield for $\left(x, \theta, x^{\prime}, \theta^{\prime}\right) \in \Gamma_{+} \times \Gamma_{-}$:

$$
\left[\tilde{\beta}^{R}-\beta^{R}\right]\left(x+\tau_{+}(x, \theta) \theta, \theta, x^{\prime}-\tau_{-}\left(x^{\prime}, \theta^{\prime}\right) \theta^{\prime}, \theta^{\prime}\right)=[\tilde{\beta}-\beta]\left(x, \theta, x^{\prime}, \theta^{\prime}\right) .
$$

The isometric relation (19) now follows from (32) and (34).

\section{THE FORWARD PROBLEM IN THREE OR HIGHER DIMENSIONS}

In this section we recall the properties which define the albedo operator and its kernel's singular expansion when $n \geq 3$.

Let $T$ be the operator defined by the left hand side of (11) considered on $B_{R} \times S^{n-1}$, $n \geq 3$. From (6), the second and the third terms of $T$ are bounded operators on $L^{1}\left(B_{R} \times S^{n-1}\right)$, while the first term is unbounded. We view $T$ as a (closed) unbounded operator on $L^{1}\left(B_{R} \times S^{n-1}\right)$ with the domain

$$
D(T)=\left\{u \in L^{1}\left(B_{R} \times S^{n-1}\right) ; \theta \cdot \nabla u \in L^{1}\left(B_{R} \times S^{n-1}\right),\left.u\right|_{\Gamma_{-}}=0\right\} ;
$$

see 12 .

We work under either one of the following subcritical conditions that yield wellposedness for the boundary value problem (11) and (3):

$$
\operatorname{ess} \sup _{(x, \theta) \in \Omega \times S^{n-1}}\left|\tau(x, \theta) \int_{S^{n-1}} k\left(x, \theta, \theta^{\prime}\right) d \theta^{\prime}\right|<1,
$$

or

$$
a(x, \theta)-\int_{S^{n-1}} k\left(x, \theta, \theta^{\prime}\right) d \theta^{\prime} \geq 0, \text { a.e. } \Omega \times S^{n-1}
$$

see, e.g., 17, 12, 13, 24, 25.

Let $\delta_{\{x\}}\left(x^{\prime}\right)$ represent the delta distribution with respect to the boundary measure $d \mu\left(x^{\prime}\right)$ supported at $x \in \partial \Omega$, and let $\delta_{\{\theta\}}\left(\theta^{\prime}\right)$ represent the delta distribution with respect to $d \theta$ centered at $\theta \in S^{n-1}$. The following result is a recast of [12, Theorem 2.3] to the unit speed velocities.

Proposition 3.1. Let $n \geq 3$. Assume that the direct problem is well-posed. Then the albedo operator $\mathcal{A}: L^{1}\left(\Gamma_{-}^{R}, d \xi\right) \rightarrow L^{1}\left(\Gamma_{+}^{R}, d \xi\right)$ is bounded and its Schwartz kernel $\alpha\left(x, \theta, x^{\prime}, \theta^{\prime}\right)$, considered as a distribution on $\Gamma_{+}$with $\left(x^{\prime}, \theta^{\prime}\right) \in \Gamma_{-}^{R}$ parameters, is given by $\alpha=\alpha_{1}+\alpha_{2}+\alpha_{3}$, where

$$
\begin{aligned}
\alpha_{1}\left(x, \theta, x^{\prime}, \theta^{\prime}\right)= & \frac{\left|n\left(x^{\prime}\right) \cdot \theta^{\prime}\right|}{n(x) \cdot \theta} e^{-\int_{0}^{\tau_{-}(x, \theta)} a(x-t \theta, \theta) d t} \delta_{\left\{x^{\prime}+\tau_{+}\left(x^{\prime}, \theta^{\prime}\right) \theta^{\prime}\right\}}(x) \delta_{\left\{\theta^{\prime}\right\}}(\theta) \\
\alpha_{2}\left(x, \theta, x^{\prime}, \theta^{\prime}\right)= & \frac{\left|n\left(x^{\prime}\right) \cdot \theta^{\prime}\right|}{n(x) \cdot \theta} \int_{0}^{\tau_{+}\left(x^{\prime}, \theta^{\prime}\right)} e^{-\int_{0}^{\tau_{+}\left(x^{\prime}+t \theta^{\prime}, \theta\right)} a(x-s \theta, \theta) d s} e^{-\int_{0}^{t} a\left(x^{\prime}+s \theta^{\prime}, \theta^{\prime}\right) d s} \\
& \times k\left(x^{\prime}+t \theta^{\prime}, \theta^{\prime}, \theta\right) \delta_{\left\{x^{\prime}+t \theta^{\prime}+\tau_{+}\left(x^{\prime}+t \theta^{\prime}, \theta\right) \theta\right\}}(x) d t \\
\left|n\left(x^{\prime}\right) \cdot \theta^{\prime}\right|^{-1} \alpha_{3} \in & L^{\infty}\left(\Gamma_{-}^{R} ; L^{1}\left(\Gamma_{+}^{R}, d \xi\right)\right) .
\end{aligned}
$$




\section{Preliminary estimates}

This section extends a result from [7] for continuous coefficients to the class of essentially bounded coefficients as in (6) . This extension is crucial to the transportation of the problem to a larger domain, if no boundary knowledge of the coefficients is available, and has the added benefit of simplifying the proof considerably.

Although the presentation below concerns the unit speed velocity and measurements at the boundary, the results easily extend to the variable velocity and measurements in the rotating planes setting. The main novelty in this section is made possible by the following result on the approximation of the identity.

Proposition 4.1. [14, Theorem 8.15] Suppose $\varphi \in L^{1}\left(\mathbb{R}^{n}\right)$ with $\int \varphi(x) d x=1$ and $|\varphi(x)| \leq C(1+|x|)^{-n-\varepsilon}$ for some $C, \varepsilon>0$, and let $\varphi_{t}=t^{-n} \varphi(x / t)$. If $f \in L^{p}\left(\mathbb{R}^{n}\right)$, for some $1 \leq p \leq \infty$, then $f * \varphi_{t} \rightarrow f(x)$ with $t \rightarrow 0^{+}$, for almost every $x$ in the Lebesgue set of $f$-in particular, for almost every $x \in \mathbb{R}^{n}$, and at every $x$ at which $f$ is continuous.

Corollary 4.2. There is a family of maps $\phi_{\varepsilon, x_{0}^{\prime}, \theta_{0}^{\prime}} \in L^{1}\left(\Gamma_{-}, d \xi\right),\left(x_{0}^{\prime}, \xi_{0}^{\prime}\right) \in \Gamma_{-}$and $\varepsilon>0$, such that, for any $f \in L^{\infty}\left(\Gamma_{+}, d \xi\right)$ given,

$$
\lim _{\varepsilon \rightarrow 0} \int_{\Gamma_{-}} \phi_{\varepsilon, x_{0}^{\prime}, \theta_{0}^{\prime}}\left(x^{\prime}, \theta^{\prime}\right) f\left(x^{\prime}, \theta^{\prime}\right) d \xi\left(x^{\prime}, \theta^{\prime}\right)=f\left(x_{0}^{\prime}, \theta_{0}^{\prime}\right),
$$

whenever $\left(x_{0}^{\prime}, \theta_{0}^{\prime}\right)$ is in the Lebesgue set of $f$. In particular, (40) holds for almost every $\left(x_{0}^{\prime}, \theta_{0}^{\prime}\right) \in \Gamma_{-}$.

Proof. For $\left(x_{0}^{\prime}, \theta_{0}^{\prime}\right) \in \Gamma_{-}$and $\varepsilon>0$ sufficiently small, let $\left(x^{\prime}, \theta^{\prime}\right): U \times V \subset \mathbb{R}^{2 n-2} \rightarrow$ $x^{\prime}(U) \times \theta^{\prime}(V) \subset \partial \Omega \times S^{n-1}$ be a coordinate chart with $\left(x_{0}^{\prime}, \theta_{0}^{\prime}\right) \in x^{\prime}(U) \times \theta^{\prime}(U)$ such that $x_{0}^{\prime}=x^{\prime}(0)$ and $\theta_{0}^{\prime}=\theta^{\prime}(0)$. For $\left(x^{\prime}, \theta^{\prime}\right) \in \Gamma_{-}$, define

$$
\phi_{\varepsilon, x_{0}^{\prime}, \theta_{0}^{\prime}}\left(x^{\prime}, \theta^{\prime}\right)=\frac{1}{\left|n\left(x^{\prime}\right) \cdot \theta^{\prime}\right|}\left|\frac{D(u, v)}{D\left(x^{\prime}, \theta^{\prime}\right)}\right| \varphi_{\varepsilon}\left(u\left(x^{\prime}\right)\right) \varphi_{\varepsilon}\left(v\left(\theta^{\prime}\right)\right),
$$

where $\varphi(u) \equiv 1 /\left(\omega_{n-1}\right)$ for $|u|<1, \varphi(u) \equiv 0$ for $|u| \geq 1$, and $\varphi_{\varepsilon}(u)=\varepsilon^{-n+1} \varphi(u / \varepsilon)$. By $\omega_{n-1}$ we denoted the volume of the $(n-1)$-dimensional unit ball. Then, for any $\varepsilon>0, \int \varphi_{\varepsilon}(u) d u=1$ and

$$
\int_{\Gamma_{-}} \phi_{\varepsilon, x_{0}^{\prime}, \theta_{0}^{\prime}}\left(x^{\prime}, \theta^{\prime}\right) f\left(x^{\prime}, \theta^{\prime}\right) d \xi\left(x^{\prime}, \theta^{\prime}\right)=\int_{R^{2 n-2}} \varphi_{\varepsilon}(u) \varphi_{\varepsilon}(v) f\left(x^{\prime}(u), \theta^{\prime}(v)\right) d u d v .
$$

Apply the equality above to $f \equiv 1$ to get $\left\|\phi_{\varepsilon, x_{0}^{\prime}, \theta_{0}^{\prime}}\right\|_{L^{1}\left(\Gamma_{+}, d \xi\right)}=1$. The conclusion follows from Proposition 4.1 .

We introduce the following notations. For $\left(x^{\prime}, \theta^{\prime}\right) \in \Gamma_{-}, 0 \leq t \leq \tau_{+}\left(x^{\prime}, \theta^{\prime}\right)$ and $\theta \in S^{n-1}$ let

$$
\begin{aligned}
x^{+}\left(t, x^{\prime}, \theta^{\prime}, \theta\right) & :=x^{\prime}+t \theta^{\prime}+\tau_{+}\left(x^{\prime}+t \theta^{\prime}, \theta\right) \theta, \\
F\left(t, x^{\prime}, \theta^{\prime}, \theta\right) & :=e^{-\int_{0}^{\tau_{+}\left(x^{\prime}+t \theta^{\prime}, \theta\right)} a\left(x^{\prime}+t \theta^{\prime}+s \theta, \theta\right) d s} e^{-\int_{0}^{t} a\left(x^{\prime}+s \theta^{\prime}, \theta^{\prime}\right) d s,}
\end{aligned}
$$

denote the exiting point after one scattering at the point $x^{\prime}+t \theta^{\prime}$ coming from direction $\theta^{\prime}$ into the direction $\theta$, and, respectively, the total absorption along the broken line 
due to this scattering. We note here that $x^{+}$depends on the point $x^{\prime}+t \theta^{\prime}$ where the scattering takes place and not explicitly on $t$. This remark will be used later in (56).

Let $(a, k),(\tilde{a}, \tilde{k})$ be as in (6) extended by 0 on $B_{R} \backslash \bar{\Omega}$, such that the forward problem is well-posed. All the operators bearing the tilde refer to $(\tilde{a}, \tilde{k})$ and are defined in a similar way with the ones for $(a, k)$. For example $\tilde{\mathcal{A}}$ is the albedo operator corresponding to $(\tilde{a}, \tilde{k})$. Recall that $n$ is the dimension of the space.

Theorem 4.3. Let $(a, k),(\tilde{a}, \tilde{k})$ be as in ([6) . For almost every $\left(x_{0}^{\prime}, \theta_{0}^{\prime}\right) \in \Gamma_{-}$the following estimates hold:

For $n \geq 2$,

$$
\left|e^{-\int_{0}^{\tau_{+}\left(x_{0}^{\prime}, \theta_{0}^{\prime}\right)} a\left(x_{0}^{\prime}+t \theta_{0}^{\prime}, \theta_{0}^{\prime}\right) d s}-e^{-\int_{0}^{\tau_{+}\left(x_{0}^{\prime}, \theta_{0}^{\prime}\right)} \tilde{a}\left(x_{0}^{\prime}+t \theta_{0}^{\prime}, \theta_{0}^{\prime}\right) d s}\right| \leq\|\mathcal{A}-\tilde{\mathcal{A}}\| .
$$

For $n \geq 3$,

$$
\begin{aligned}
\int_{0}^{\tau_{+}\left(x_{0}^{\prime}, \theta_{0}^{\prime}\right)} \int_{S^{n-1}} \mid k & -\tilde{k} \mid\left(x_{0}^{\prime}+t \theta_{0}^{\prime}, \theta^{\prime}, \theta\right) E\left(t, x_{0}^{\prime}, \theta_{0}^{\prime}, \theta\right) d \theta d t \leq\|\mathcal{A}-\tilde{\mathcal{A}}\|+ \\
& +\|F-\tilde{F}\|_{\infty} \int_{0}^{\tau_{+}\left(x_{0}^{\prime}, \theta_{0}^{\prime}\right)} \int_{S^{n-1}} \tilde{k}\left(x_{0}+t \theta_{0}^{\prime}, \theta_{0}^{\prime}, \theta\right) d \theta d t .
\end{aligned}
$$

Proof. Let $\left(x_{0}^{\prime}, \theta_{0}^{\prime}\right) \in \Gamma_{-}$be arbitrarily fixed and let $\phi_{\varepsilon, x_{0}^{\prime}, \theta_{0}^{\prime}} \in L^{1}\left(\Gamma_{-}\right)$be defined as in Corollary 4.2. To simplify the formulas, since $\left(x_{0}^{\prime}, \theta_{0}^{\prime}\right)$ is fixed, in the following we drop this dependence from the notation $\phi_{\varepsilon}=\phi_{\varepsilon, x_{0}^{\prime}, \theta_{0}^{\prime}}$.

Let $\mathcal{A}=\mathcal{A}_{1}+\mathcal{A}_{2}+\mathcal{A}_{3}$ be the decomposition of the albedo operator given by

$$
\mathcal{A}_{i} f(x, \theta)=\int_{\Gamma_{-}} \alpha_{i}\left(x, \theta, x^{\prime}, \theta^{\prime}\right) f\left(x^{\prime}, \theta^{\prime}\right) d \mu\left(x^{\prime}\right) d \theta^{\prime}, i=1,2,3,
$$

where $\alpha_{i}, i=1,2,3$ are the Schwartz kernels in Proposition 3.1 and $d \mu\left(x^{\prime}\right)$ is the induced Lebesgue measure on the boundary $\partial \Omega$.

Let $\phi \in L^{\infty}\left(\Gamma_{+}\right)$with $\|\phi\|_{\infty} \leq 1$. Since $\left\|\phi_{\varepsilon}\right\|_{L^{1}\left(\Gamma_{-}\right)}=1$, the mapping properties of the albedo operator imply that

$$
\left|\int_{\Gamma_{+}} \phi(x, \theta)[\mathcal{A}-\tilde{\mathcal{A}}] \phi_{\varepsilon}(x, \theta) d \xi(x, \theta)\right| \leq\|\mathcal{A}-\tilde{\mathcal{A}}\| .
$$

Next we evaluate each of the three terms in $\int_{\Gamma_{+}} \phi(x, \theta)[\mathcal{A}-\tilde{\mathcal{A}}] \phi_{\varepsilon}(x, \theta) d \xi(x, \theta)$ by using the decomposition in Proposition 3.1 and Fubini's theorem.

The first term is evaluated using the formula (37):

$$
\begin{aligned}
I_{1}(\phi, \varepsilon):=\int_{\Gamma_{+}} \phi(x, \theta) & {\left[\mathcal{A}_{1}-\tilde{\mathcal{A}}_{1}\right] \phi_{\varepsilon}(x, \theta) d \xi(x, \theta)=\int_{\Gamma_{-}} \phi\left(x^{\prime}+\tau_{+}\left(x^{\prime}, \theta^{\prime}\right), \theta^{\prime}\right) \phi_{\varepsilon}\left(x^{\prime}, \theta^{\prime}\right) } \\
& \times\left[e^{-\int_{0}^{\tau_{+}\left(x^{\prime}, \theta^{\prime}\right)} a\left(x^{\prime}+s \theta^{\prime}, \theta^{\prime}\right) d s}-e^{-\int_{0}^{\tau_{+}\left(x^{\prime}, \theta^{\prime}\right)} \tilde{a}\left(x^{\prime}+s \theta^{\prime}, \theta^{\prime}\right) d s}\right] d \xi\left(x^{\prime}, \theta^{\prime}\right) .
\end{aligned}
$$


Since the integrand above is in $L^{\infty}\left(\Gamma_{-}\right)$by applying (40), we get for almost every $\left(x_{0}^{\prime}, \theta_{0}^{\prime}\right) \in \Gamma_{-}$

$I_{1}(\phi)\left(x_{0}^{\prime}, \theta_{0}^{\prime}\right):=\lim _{\varepsilon \rightarrow 0} I_{1}(\phi, \varepsilon)$

$$
=\phi\left(x_{0}^{\prime}+\tau_{+}\left(x_{0}^{\prime}, \theta_{0}^{\prime}\right), \theta_{0}^{\prime}\right)\left(e^{-\int_{0}^{\tau_{+}\left(x_{0}^{\prime}, \theta_{0}^{\prime}\right)} a\left(x_{0}^{\prime}+s \theta_{0}^{\prime}, \theta_{0}^{\prime}\right) d s}-e^{-\int_{0}^{\tau_{+}\left(x_{0}^{\prime}, \theta_{0}^{\prime}\right)} \tilde{a}\left(x_{0}^{\prime}+s \theta_{0}^{\prime}, \theta_{0}^{\prime}\right) d s}\right) .
$$

To evaluate the second term we use the notations (42) and (43) and the formula (38):

$$
\begin{aligned}
I_{2}(\phi, \varepsilon) & :=\int_{\Gamma_{+}} \phi(x, \theta)\left[\mathcal{A}_{2}-\tilde{\mathcal{A}}_{2}\right] \phi_{\varepsilon}(x, \theta) d \xi(x, \theta) \\
& =\int_{\Gamma_{-}} \phi_{\varepsilon}\left(x^{\prime}, \theta^{\prime}\right) d \xi\left(x^{\prime}, \theta^{\prime}\right)\left\{\int_{S^{n-1}} \int_{0}^{\tau_{+}\left(x^{\prime}, \theta^{\prime}\right)} \phi\left(x^{+}\left(t, x^{\prime}, \theta^{\prime}, \theta\right), \theta\right) \times\right. \\
& \left.\times\left[F\left(t, x^{\prime}, \theta^{\prime}, x, \theta\right) k\left(x^{\prime}+t \theta^{\prime}, \theta^{\prime}, \theta\right)-\tilde{F}\left(t, x^{\prime}, \theta^{\prime}, x, \theta\right) \tilde{k}\left(x^{\prime}+t \theta^{\prime}, \theta^{\prime}, \theta\right)\right] d t d \theta\right\} .
\end{aligned}
$$

Apply again (40) for the continuous integrand above to obtain for almost every $\left(x_{0}^{\prime}, \theta_{0}^{\prime}\right) \in \Gamma_{-}$:

$$
\begin{aligned}
I_{2}(\phi)\left(x_{0}^{\prime}, \theta_{0}^{\prime}\right) & :=\lim _{\varepsilon \rightarrow 0} I_{2}(\phi, \varepsilon) \\
& =\int_{S^{n-1}} \int_{0}^{\tau_{+}\left(x_{0}^{\prime}, \theta_{0}^{\prime}\right)} \phi\left(x^{+}\left(t, x_{0}^{\prime}, \theta_{0}^{\prime}, \theta\right), \theta\right)\left[F\left(t, x_{0}^{\prime}, \theta_{0}^{\prime}, x, \theta\right) k\left(x_{0}^{\prime}+t \theta_{0}^{\prime}, \theta_{0}^{\prime}, \theta\right)\right. \\
& \left.-\tilde{F}\left(t, x_{0}^{\prime}, \theta_{0}^{\prime}, x, \theta\right) \tilde{k}\left(x_{0}^{\prime}+t \theta_{0}^{\prime}, \theta_{0}^{\prime}, \theta\right)\right] d t d \theta,
\end{aligned}
$$

or $I_{2}(\phi)=I_{2,1}(\phi)+I_{2,2}(\phi)$ with

$$
I_{2,1}(\phi)=\int_{S^{n-1}} \int_{0}^{\tau_{+}\left(x_{0}^{\prime}, \theta_{0}^{\prime}\right)} \phi\left(x^{+}\left(t, x_{0}^{\prime}, \theta_{0}^{\prime}, \theta\right), \theta\right) F\left(t, x_{0}^{\prime}, \theta_{0}^{\prime}, x, \theta\right)(k-\tilde{k})\left(x_{0}^{\prime}+t \theta_{0}^{\prime}, \theta_{0}^{\prime}, \theta\right) d t d \theta,
$$

$$
\left|I_{2,2}(\phi)\right| \leq \int_{S^{n-1}} \int_{0}^{\tau_{+}\left(x_{0}^{\prime}, \theta_{0}^{\prime}\right)}|F-\tilde{F}|\left(t, x_{0}^{\prime}, \theta_{0}^{\prime}, x, \theta\right) \tilde{k}\left(x_{0}^{\prime}+t \theta_{0}^{\prime}, \theta_{0}^{\prime}, \theta\right) d t d \theta
$$

Consider the third term

$$
\begin{aligned}
I_{3}(\phi, \varepsilon) & =\int_{\Gamma_{+}} \phi(x, \theta)\left[\mathcal{A}_{3}-\tilde{\mathcal{A}}_{3}\right] \phi_{\varepsilon}(x, \theta) d \xi(x, \theta) \\
& =\int_{\Gamma_{-}} \phi_{\varepsilon}\left(x^{\prime}, \theta^{\prime}\right) d \xi\left(x^{\prime}, \theta^{\prime}\right)\left\{\int_{\Gamma_{+}} \phi(x, \theta) \frac{\left(\alpha_{3}-\tilde{\alpha}_{3}\right)\left(x, \theta, x^{\prime}, \theta^{\prime}\right)}{\left|n\left(x^{\prime}\right) \cdot \theta^{\prime}\right|} d \xi(x, \theta)\right\} .
\end{aligned}
$$

By (39), the map $\left(x^{\prime}, \theta^{\prime}\right) \mapsto \int_{\Gamma_{+}} \phi(x, \theta)\left(\alpha_{3}-\tilde{\alpha}_{3}\right)\left(x, \theta, x^{\prime}, \theta^{\prime}\right)\left|n\left(x^{\prime}\right) \cdot \theta^{\prime}\right|^{-1} d \xi(x, \theta)$ is in $L^{\infty}\left(\Gamma_{-}\right)$, and then, by (40) we get for almost every $\left(x_{0}^{\prime}, \theta_{0}^{\prime}\right) \in \Gamma_{-}$

$$
I_{3}(\phi)\left(x_{0}^{\prime}, \theta_{0}^{\prime}\right):=\lim _{\varepsilon \rightarrow 0} I_{3}(\phi, \varepsilon)=\int_{\Gamma_{+}} \phi(x, \theta) \frac{\left|\alpha_{3}-\tilde{\alpha}_{3}\right|\left(x, \theta, x_{0}^{\prime}, \theta_{0}^{\prime}\right)}{\left|n\left(x_{0}^{\prime}\right) \cdot \theta_{0}^{\prime}\right|} d \xi(x, \theta) .
$$


The left hand side of (46) has three terms. We move the third term to the right hand side (with absolute values) and take the limit with $\varepsilon \rightarrow 0$ to get

$$
\left|I_{1}(\phi)+I_{2}(\phi)\right|\left(x_{0}^{\prime}, \theta_{0}^{\prime}\right) \leq\|\mathcal{A}-\tilde{\mathcal{A}}\|+I_{3}(|\phi|)\left(x_{0}^{\prime}, \theta_{0}^{\prime}\right), \text { a.e. }\left(x_{0}^{\prime}, \theta_{0}^{\prime}\right) \in \Gamma_{-},
$$

for any $\phi \in L^{\infty}\left(\Gamma_{+}\right)$with $\|\phi\|_{\infty}=1$.

We note that the negligible set on which the inequality above does not hold may depend on $\phi$. In (53), we shall choose two sequences of $\phi$ to conclude the estimates of the lemma. Since countable union of negligible sets is negligible, the inequality (53) holds almost everywhere on $\Gamma_{-}$, independently of the term in the sequence. This justifies the argument below for almost every $\left(x_{0}^{\prime}, \theta_{0}^{\prime}\right)$ in $\Gamma_{-}$.

First we show the estimate (44). Let $\left\{\phi_{m}\right\}$ be a sequence of maps such that $\left|\phi_{m}\right| \leq 1, \phi_{m} \equiv 1$ near $\left(x_{0}^{\prime}+\tau_{+}\left(x_{0}^{\prime}, \theta_{0}^{\prime}\right) \theta_{0}^{\prime}, \theta_{0}^{\prime}\right)$ and with support inside $\{(x, \theta) \in$ $\left.\Gamma_{+}:\left|x-x_{0}^{\prime}+\tau_{+}\left(x_{0}^{\prime}, \theta_{0}^{\prime}\right) \theta_{0}^{\prime}\right|+\left|\theta-\theta_{0}^{\prime}\right|<1 / m\right\}$. Then (48) gives

$$
I_{1}\left(\phi_{m}\right)=e^{-\int_{0}^{\tau_{+}\left(x_{0}^{\prime}, \theta_{0}^{\prime}\right)} a\left(x_{0}^{\prime}+s \theta_{0}^{\prime}, \theta_{0}^{\prime}\right) d s}-e^{-\int_{0}^{\tau_{+}\left(x_{0}^{\prime}, \theta_{0}^{\prime}\right)} \tilde{a}\left(x_{0}^{\prime}+s \theta_{0}^{\prime}, \theta_{0}^{\prime}\right) d s},
$$

independently of $m$. From (49) we have $\lim _{m \rightarrow \infty} I_{2}\left(\phi_{m}\right)=0$ since the support in $\theta$ shrinks to $\theta_{0}^{\prime}$. From (52) we also have $\lim _{n \rightarrow \infty} I_{3}\left(\left|\phi_{m}\right|\right)=0$, since the support shrinks to one point.

Next we prove the estimate (45). Let $\left\{\phi_{m, q}\right\}$ be a (double indexed) sequence defined by $\phi_{m, q}(x, \theta)=\chi_{m}(\theta) \varphi_{q}(x, \theta)$ where $\chi_{m} \in L^{\infty}\left(S^{n-1}\right)$ with $\chi_{m} \equiv 0$ for $\left|\theta-\theta_{0}^{\prime}\right| \leq 1 / m$ and $\chi_{m} \equiv 1$ for $\left|\theta-\theta_{0}^{\prime}\right|>1 / m$ and $\varphi_{q} \in L^{\infty}\left(\Gamma_{+}\right)$to be specified below. Regardless of the way we define $\varphi_{q}$, the presence of $\chi_{m}$ already yields $I_{1}\left(\phi_{m, q}\right)=0$ for all $m, q$ since $\chi_{m}\left(x_{0}^{\prime}+\tau_{+}\left(x_{0}^{\prime}, \theta_{0}^{\prime}\right) \theta_{0}^{\prime}, \theta_{0}^{\prime}\right)=0$.

Next we construct $\varphi_{q}$. Note that we only need to have it defined for $\theta \neq \theta_{0}^{\prime}$ since the function $\chi$ vanishes nearby $\theta_{0}^{\prime}$.

Define first $\varphi_{q}(x, \theta)$ as follows. Using the notation (42), let

$$
\Pi_{x_{0}^{\prime}, \theta_{0}^{\prime}, \theta}:=\left\{x^{+}\left(t, x_{0}^{\prime}, \theta_{0}^{\prime}, \theta\right) \in \partial B_{R}: 0 \leq t \leq \tau_{+}\left(x_{0}^{\prime}, \theta_{0}^{\prime}\right)\right\}
$$

represent the set of the exit points of particles entering in the direction $\theta_{0}^{\prime}$ at $x_{0}^{\prime}$ which scattered once in the direction of $\theta$. The set $\Pi_{x_{0}^{\prime}, \theta_{0}^{\prime}, \theta}$ lies in the intersection of the plane through $x_{0}^{\prime}$ spanned by $\theta_{0}^{\prime}, \theta$ and the boundary $\partial B_{R}$.

Now, for each $\theta \in S^{n-1}$ and $x^{+}\left(t, x_{0}^{\prime}, \theta_{0}^{\prime}, \theta\right)$ of (42) in the set

$$
\left\{x^{+} \in \partial B_{R}: \operatorname{dist}_{\partial B_{R}}\left(x^{+}, \Pi_{x_{0}^{\prime}, \theta_{0}^{\prime}, \theta}\right)<1 / m\right\}
$$

we define

$$
\varphi_{q}\left(x^{+}\left(t, x_{0}^{\prime}, \theta_{0}^{\prime}, \theta\right), \theta\right):=\operatorname{sgn}(k-\tilde{k})\left(x_{0}^{\prime}+t \theta_{0}^{\prime}, \theta_{0}^{\prime}, \theta\right) .
$$

Outside the set in (55) we define $\varphi_{q}(\cdot, \theta) \equiv 0$.

Since $x^{+}\left(t, x_{0}^{\prime}, \theta_{0}^{\prime}, \theta\right)$ in (42) depends only on $x_{0}^{\prime}+t \theta_{0}^{\prime}, \theta_{0}^{\prime}$ and $\theta$, the equation (56) gives a well defined function $\varphi_{q} \in L^{\infty}\left(B_{R} \times S^{n-1}\right)$. Note also that (55) shrinks to a negligible set on $\partial B_{R}$ with $q \rightarrow \infty$.

Now apply the estimate (53) to $\phi_{m, q}$ and use $I_{1}\left(\phi_{m, q}\right)=0$ to get

$$
\left|I_{2,1}\left(\phi_{m, q}\right)\right|\left(x_{0}^{\prime}, \theta_{0}^{\prime}\right) \leq\|\mathcal{A}-\tilde{\mathcal{A}}\|+I_{3}\left(\left|\phi_{m, q}\right|\right)\left(x_{0}^{\prime}, \theta_{0}^{\prime}\right)+\left|I_{2,2}\left(\phi_{m, q}\right)\right|\left(x_{0}^{\prime}, \theta_{0}^{\prime}\right) .
$$


Since the support of $\phi_{m, q}$ shrinks to a set of measure zero in $\Gamma_{+}$as $q \rightarrow \infty$, we get for all $m$ and almost every $\left(x_{0}^{\prime}, \theta_{0}^{\prime}\right) \in \Gamma_{-}, \lim _{q \rightarrow \infty} I_{3}\left(\left|\phi_{m, q}\right|\right)\left(x_{0}^{\prime}, \theta_{0}^{\prime}\right)=0$. Finally, from (50) we obtain for almost every $\left(x_{0}^{\prime}, \theta_{0}^{\prime}\right) \in \Gamma_{-}$that

$$
\lim _{m \rightarrow \infty} \lim _{n \rightarrow \infty} I_{2,1}\left(\phi_{m, q}\right)=\int_{S^{n-1}} \int_{0}^{\tau_{+}\left(x_{0}^{\prime}, \theta_{0}^{\prime}\right)} F\left(t, x_{0}^{\prime}, \theta_{0}^{\prime}, x, \theta\right)|k-\tilde{k}|\left(x_{0}^{\prime}+t \theta_{0}^{\prime}, \theta_{0}^{\prime}, \theta\right) d t d \theta,
$$

while from (51) we have

$$
\left|I_{2,2}\left(\phi_{m, q}\right)\right| \leq \int_{S^{n-1}} \int_{0}^{\tau_{+}\left(x_{0}^{\prime}, \theta_{0}^{\prime}\right)}|F-\tilde{F}|\left(t, x_{0}^{\prime}, \theta_{0}^{\prime}, x, \theta\right) \tilde{k}\left(x_{0}^{\prime}+t \theta_{0}^{\prime}, \theta_{0}^{\prime}, \theta\right) d t d \theta,
$$

for all $m, q$. The estimate (45) in the lemma follows.

\section{Stability modulo gauge transformations}

In this section we prove Theorem 1.2 .

We start with two pairs $(a, k),(\tilde{a}, \tilde{k}) \in U_{\Sigma, \rho}$ and let

$$
\varepsilon:=\|\mathcal{A}-\tilde{\mathcal{A}}\| \text {. }
$$

We shall find an intermediate pair $\left(a^{\prime}, k^{\prime}\right) \sim(a, k)$ such that (13) and (14) hold for some constant $C>0$ dependent on $\Sigma, \rho, \Omega$ and $B_{R}$.

Define first the "trial" gauge transformation:

$$
\varphi(x, \theta):=e^{-\int_{0}^{\tau-(x, \theta)}(\tilde{a}-a)(x-s \theta, \theta) d s} \text {, a.e. }(x, \theta) \in B_{R} \times S^{n-1} .
$$

Then $\varphi>0,\left.\varphi\right|_{\Gamma_{-}^{R}}=1, \theta \cdot \nabla \varphi(x, \theta) \in L^{\infty}\left(B_{R} \times S^{n-1}\right)$ and

$$
\tilde{a}(x, \theta)=a(x, \theta)-\theta \cdot \nabla_{x} \ln \varphi(x, \theta) .
$$

Note that $\left.\varphi\right|_{\Gamma_{+}^{R}}$ is close, but not equal, to 1 . We use the first estimate from Lemma 4.3 to decide how far from 1 can $\left.\varphi\right|_{\Gamma_{+}^{R}}$ be.

By (44), we have for almost every $\left(x_{0}^{\prime}, \theta_{0}^{\prime}\right) \in \Gamma_{-}$

$$
\left|e^{-\int_{0}^{\tau_{+}\left(x_{0}^{\prime}, \theta_{0}^{\prime}\right)} a\left(x_{0}^{\prime}+t \theta_{0}^{\prime}, \theta_{0}^{\prime}\right) d s}-e^{-\int_{0}^{\tau_{+}+\left(x_{0}^{\prime}, \theta_{0}^{\prime}\right)} \tilde{a}\left(x_{0}^{\prime}+t \theta_{0}^{\prime}, \theta_{0}^{\prime}\right) d s}\right| \leq \varepsilon
$$

In each of the integrals of the left hand side above change variables $t=\tau_{+}\left(x_{0}^{\prime}, \theta_{0}^{\prime}\right)-s$ and denote $x_{0}=x_{0}^{\prime}+\tau_{+}\left(x_{0}^{\prime}, \theta_{0}^{\prime}\right) \theta_{0}^{\prime}$ to get

$$
\left|e^{-\int_{0}^{\tau_{-}\left(x_{0}, \theta_{0}^{\prime}\right)} a\left(x_{0}-s \theta_{0}^{\prime}, \theta_{0}^{\prime}\right) d s}-e^{-\int_{0}^{\tau_{-}-\left(x_{0}, \theta_{0}^{\prime}\right)} \tilde{a}\left(x_{0}-s \theta_{0}^{\prime}, \theta_{0}^{\prime}\right) d s}\right| \leq \varepsilon
$$

When $\left(x_{0}^{\prime}, \theta_{0}^{\prime}\right)$ covers $\Gamma_{-}^{R}$ almost everywhere we get that $\left(x_{0}, \theta_{0}^{\prime}\right)$ covers $\Gamma_{+}^{R}$ almost everywhere. 
Now apply the Mean Value theorem to $u \mapsto e^{-u}$ to get the lower bound

$$
\begin{aligned}
\left|e^{-\int_{0}^{\tau_{-}\left(x_{0}, \theta_{0}^{\prime}\right)} a\left(x_{0}-s \theta_{0}^{\prime}, \theta_{0}^{\prime}\right) d s}-e^{-\int_{0}^{\tau_{-}\left(x_{0}, \theta_{0}^{\prime}\right)} \tilde{a}\left(x_{0}-s \theta_{0}^{\prime}, \theta_{0}^{\prime}\right) d s}\right| & \\
= & e^{-u_{0}}\left|\int_{0}^{\tau_{-}\left(x_{0}, \theta_{0}^{\prime}\right)}(\tilde{a}-a)\left(x_{0}-s \theta_{0}^{\prime}, \theta_{0}^{\prime}\right) d s\right| \\
& =e^{-u_{0}}\left|\ln \varphi\left(x_{0}, \theta_{0}^{\prime}\right)\right| \geq e^{-2 R \Sigma}\left|\ln \varphi\left(x_{0}, \theta_{0}^{\prime}\right)\right| .
\end{aligned}
$$

where $u_{0}=u_{0}\left(x_{0}, \theta_{0}^{\prime}, a, \tilde{a}\right)$ is a value between the two integrals appearing at the exponent in the left hand side above, and $\varphi$ is defined in (58).

From (60) and (61) we get the following estimate for the "trial" gauge $\varphi$ :

$$
|\ln \varphi(x, \theta)| \leq e^{2 R \Sigma} \varepsilon \text {, a.e. }(x, \theta) \in \Gamma_{+}^{R} .
$$

The "trial" gauge $\varphi$ is not good enough since it does not equal 1 on $\Gamma_{+}^{R}$. We alter it to some $\tilde{\varphi} \in L^{\infty}\left(B_{R} \times S^{1}\right)$ with $\theta \cdot \nabla \ln \tilde{\varphi} \in L^{\infty}\left(B_{R} \times S^{n-1}\right)$ in such a way that $\left.\tilde{\varphi}\right|_{\partial B_{R}}=1$. More precisely, for almost every $(x, \theta) \in \bar{B}_{R} \times S^{n-1}$, we define $\tilde{\varphi}(x, \theta)$ by

$$
\ln \tilde{\varphi}(x, \theta):=\ln \varphi(x, \theta)-\frac{\tau_{-}(x, \theta)}{\tau(x, \theta)} \ln \varphi\left(x+\tau_{+}(x, \theta) \theta, \theta\right) .
$$

Since $0 \leq \tau_{-}(x, \theta) / \tau(x, \theta) \leq 1$ we get $\tilde{\varphi} \in L^{\infty}\left(B_{R} \times S^{1}\right)$. Following directly from its definition $\left.\ln \tilde{\varphi}\right|_{\partial B_{R}}=0$ : Indeed, for $(x, \theta) \in \Gamma_{-}^{R}$, we get that $\tau_{-}(x, \theta)=0$ and $\varphi(x, \theta)=$ 1 , whereas for $(x, \theta) \in \Gamma_{+}^{R}$ we have that $\tau_{-}(x, \theta)=\tau(x, \theta)$ and $x=x+\tau_{+}(x, \theta) \theta$. Since both maps $x \mapsto \tau(x, \theta)$ and $x \mapsto \ln \varphi\left(x+\tau_{+}(x, \theta) \theta, \theta\right)$ are constant in the direction of $\theta$ and since $\theta \cdot \nabla_{x} \tau_{-}(x, \theta)=1$, we get $\theta \cdot \nabla \ln \tilde{\varphi}(x, \theta) \in L^{\infty}\left(B_{R} \times S^{n-1}\right)$ and

$$
\theta \cdot \nabla \ln \tilde{\varphi}(x, \theta)=\theta \cdot \nabla \ln \varphi(x, \theta)-\frac{\ln \varphi\left(x+\tau_{+}(x, \theta) \theta, \theta\right)}{\tau(x, \theta)} .
$$

Define now the pair $\left(a^{\prime}, k^{\prime}\right)$ in the equivalence class of $\langle a, k\rangle$ by

$$
\begin{aligned}
a^{\prime}(x, \theta) & :=a(x, \theta)-\theta \cdot \nabla_{x} \ln \tilde{\varphi}(x, \theta), \\
k^{\prime}\left(x, \theta^{\prime}, \theta\right) & :=\frac{\tilde{\varphi}(x, \theta)}{\tilde{\varphi}\left(x^{\prime}, \theta^{\prime}\right)} k\left(x, \theta^{\prime}, \theta\right) .
\end{aligned}
$$

Now $\mathcal{A}^{\prime}$, the albedo operator corresponding to $\left(a^{\prime}, k^{\prime}\right)$, satisfies $\mathcal{A}^{\prime}=\mathcal{A}$, and then

$$
\left\|\mathcal{A}^{\prime}-\tilde{\mathcal{A}}\right\|=\|\mathcal{A}-\tilde{\mathcal{A}}\|=\varepsilon \text {. }
$$

Next we compare the pairs $\left(a^{\prime}, k^{\prime}\right)$ with $(\tilde{a}, \tilde{k})$ and show them to satisfy (13) and (14).

Using the definitions (159), (65), the relation (64), and the estimate for $\varphi$ on $\Gamma_{+}^{R}$ (62), we have for almost every $(x, \theta) \in B_{R} \times S^{n-1}$ :

$$
\begin{aligned}
\left|\tilde{a}(x, \theta)-a^{\prime}(x, \theta)\right| & =\left|[\tilde{a}-a](x, \theta)+\left[a-a^{\prime}\right](x, \theta)\right| \\
& =\left|\theta \cdot \nabla_{x} \ln \tilde{\varphi}(x, \theta)-\theta \cdot \nabla_{x} \ln \varphi(x, \theta)\right| \\
& =\frac{\left|\ln \varphi\left(x+\tau_{+}(x, \theta) \theta, \theta\right)\right|}{\tau(x, \theta)} \leq \varepsilon \frac{e^{2 R \Sigma}}{\tau(x, \theta)} .
\end{aligned}
$$


Since the coefficients are supported away from $\partial B_{R}$ (by construction of $B_{R}$ ) such that (10) holds, following (68) we obtain the estimate (13) in the form

$$
\left\|\tilde{a}-a^{\prime}\right\|_{\infty} \leq \varepsilon \frac{e^{2 R \Sigma}}{c_{R}},
$$

with $c_{R}$ from (10).

Up to this point, all the arguments above also work for two dimensional domains.

Next we prove the estimate (14). These arguments are specific to three or higher dimensions. Recall the formula (43) adapted to $a^{\prime}$

$$
F^{\prime}\left(t, x^{\prime}, \theta^{\prime}, \theta\right)=e^{-\int_{0}^{\tau_{+}\left(x^{\prime}+t \theta^{\prime}, \theta\right)} a^{\prime}\left(x^{\prime}+t \theta^{\prime}+s \theta, \theta\right) d s} e^{-\int_{0}^{t} a^{\prime}\left(x^{\prime}+s \theta^{\prime}, \theta^{\prime}\right) d s}
$$

and note that $F=F^{\prime}$ is a quantity preserved under the gauge transformation (65). This follows by direct calculation and the fact that $\tilde{\varphi}=1$ on $\partial B_{R}$. Then for almost all $\left(x^{\prime}, \theta^{\prime}\right) \in \Gamma_{-}^{R}, t \in\left[0, \tau\left(x^{\prime}, \theta^{\prime}\right)\right]$ and $\theta \in S^{n-1}$, we have the following lower bound

$$
\left|F^{\prime}\left(t, x^{\prime}, \theta^{\prime}, \theta\right)\right| \geq e^{-4 R \Sigma} \text {. }
$$

Using the non-negativity of $\tilde{a}$ and $a^{\prime}$ we estimate

$$
\begin{aligned}
\mid\left[\tilde{F}-F^{\prime}\right] & \left(t, x^{\prime}, \theta^{\prime}, \theta\right)|\leq| e^{-\int_{0}^{t} \tilde{a}\left(x^{\prime}+s \theta^{\prime}, \theta^{\prime}\right) d s}-e^{\int_{0}^{t} a^{\prime}\left(x^{\prime}+s \theta^{\prime}, \theta^{\prime}\right) d s} \mid \\
& +\left|e^{-\int_{0}^{\tau_{+}\left(x^{\prime}+t \theta^{\prime}, \theta\right)} \tilde{a}\left(x^{\prime}+t \theta^{\prime}+s \theta, \theta\right) d s}-e^{-\int_{0}^{\tau_{+}\left(x^{\prime}+t \theta^{\prime}, \theta\right)} a^{\prime}\left(x^{\prime}+t \theta^{\prime}+s \theta, \theta\right) d s}\right| \\
& \leq\left|\int_{0}^{t}\left[\tilde{a}-a^{\prime}\right]\left(x^{\prime}+s \theta^{\prime}, \theta^{\prime}\right) d s\right|+\left|\int_{0}^{\tau_{+}\left(x^{\prime}+t \theta^{\prime}, \theta\right)}\left[\tilde{a}-a^{\prime}\right]\left(x^{\prime}+t \theta^{\prime}+s \theta, \theta\right) d s\right| \\
& \leq \varepsilon e^{2 R \Sigma}\left(\int_{0}^{t} \frac{d s}{\tau\left(x^{\prime}+s \theta^{\prime}, \theta^{\prime}\right)}+\int_{0}^{\tau_{+}\left(x^{\prime}+t \theta^{\prime}, \theta\right)} \frac{d s}{\tau\left(x^{\prime}+t \theta^{\prime}+s \theta, \theta\right)}\right) \\
& =\varepsilon e^{2 R \Sigma}\left(\frac{t}{\tau\left(x^{\prime}, \theta^{\prime}\right)}+\frac{\tau_{+}\left(x^{\prime}+t \theta^{\prime}, \theta\right)}{\tau\left(x^{\prime}+t \theta^{\prime}, \theta\right)}\right) \leq 2 \varepsilon e^{2 R \Sigma} .
\end{aligned}
$$

The next to the last inequality uses (68); the following equality uses the fact that both maps $s \mapsto \tau\left(x^{\prime}+s \theta^{\prime}, \theta^{\prime}\right)$ and $s \mapsto \tau\left(x^{\prime}+t \theta^{\prime}+s \theta, \theta\right)$ are constant in $s$, while the last inequality uses the fact that $t \leq \tau\left(x^{\prime}, \theta^{\prime}\right)$ and $\tau_{+}\left(x^{\prime}+t \theta^{\prime}+s \theta, \theta\right) \leq \tau\left(x^{\prime}+t \theta^{\prime}+s \theta, \theta\right)$. Therefore we proved that for almost all $\left(x^{\prime}, \theta^{\prime}\right) \in \Gamma_{-}, t \in\left[0, \tau\left(x^{\prime}, \theta^{\prime}\right)\right]$ and $\theta \in S^{n-1}$ we have

$$
\left|\left[\tilde{F}-F^{\prime}\right]\left(t, x^{\prime}, \theta^{\prime}, \theta\right)\right| \leq 2 \varepsilon e^{2 R \Sigma} .
$$

Recall now the estimate (45) with respect to the pairs $\left(a^{\prime}, k^{\prime}\right)$ and $(\tilde{a}, \tilde{k})$ :

$$
\begin{array}{r}
\int_{0}^{\tau_{+}\left(x_{0}^{\prime}, \theta_{0}^{\prime}\right)} \int_{S^{n-1}}\left|\tilde{k}-k^{\prime}\right|\left(x_{0}^{\prime}+t \theta_{0}^{\prime}, \theta^{\prime}, \theta\right) F^{\prime}\left(t, x_{0}^{\prime}, \theta_{0}^{\prime}, \theta\right) d \theta d t \leq\left\|\tilde{\mathcal{A}}-\mathcal{A}^{\prime}\right\|+ \\
+\left\|\tilde{F}-F^{\prime}\right\|_{\infty} \int_{0}^{\tau_{+}\left(x_{0}^{\prime}, \theta_{0}^{\prime}\right)} \int_{S^{n-1}} \tilde{k}\left(x_{0}+t \theta_{0}^{\prime}, \theta_{0}^{\prime}, \theta\right) d \theta d t .
\end{array}
$$


Now use the lower bound for $F^{\prime}$ in (70), the upper bound for $\left\|\tilde{F}-F^{\prime}\right\|_{\infty}$ in (72) and the hypothesis $\|\tilde{k}\|_{\infty, 1} \leq \rho$, to obtain

$$
\int_{0}^{\tau_{+}\left(x_{0}^{\prime}, \theta_{0}^{\prime}\right)} \int_{S^{n-1}}\left|\tilde{k}-k^{\prime}\right|\left(x_{0}^{\prime}+t \theta_{0}^{\prime}, \theta^{\prime}, \theta\right) d \theta d t \leq \varepsilon e^{4 R \Sigma}\left(1+2 \rho e^{2 R \Sigma}\right) .
$$

Finally, integrating the formula above in $\left(x_{0}^{\prime}, \theta_{0}^{\prime}\right) \in \Gamma_{-}$with the measure $d \xi\left(x_{0}^{\prime}, \theta_{0}^{\prime}\right)$, we get

$$
\left\|\tilde{k}-k^{\prime}\right\|_{1} \leq \varepsilon \pi R e^{4 R \Sigma}\left(1+2 \rho e^{2 R \Sigma}\right) .
$$

Now choose

$$
C=\max \left\{\pi R e^{4 R \Sigma}\left(1+2 \rho e^{2 R \Sigma}\right), e^{2 R \Sigma} / c_{R}\right\}
$$

with $c_{R}$ from (10) to finish the proof of Theorem 1.2.

\section{Preliminaries FOR TWO Dimensional DOMAins}

This section introduces the framework for the problem in two dimensions. The results are mainly from [29].

As above, $T$ denotes the operator defined by the left hand side of (1) in $B_{R} \times S^{1}$ with $B_{R} \subset \mathbb{R}^{2}$. The coefficients are extended to be 0 in $B_{R} \backslash \Omega$. By the regularity assumption (17), the second and the third terms of $T$ are bounded operators in $L^{\infty}\left(B_{R} \times S^{1}\right)$. The first term is unbounded. We view $T$ as a (closed) unbounded operator on $L^{\infty}\left(B_{R} \times S^{1}\right)$ with the domain

$$
D(T)=\left\{u \in L^{\infty}\left(B_{R} \times S^{1}\right) ; \theta \cdot \nabla u \in L^{\infty}\left(B_{R} \times S^{1}\right),\left.u\right|_{\Gamma_{-}^{R}} \in L^{\infty}\left(\Gamma_{-}^{R}\right)\right\} .
$$

To simplify notation, for $x \neq y$, we denote by

$$
\widehat{x-y}=\arg (x-y)=\frac{x-y}{|x-y|},
$$

the direction from $y$ to $x$. Also, let

$$
0<E(y, x)=e^{-\int_{0}^{1} a(x-t(x-y) ; \widehat{x-y}) d t} \leq 1
$$

denote the attenuation along the segment in the direction from $y$ to $x$. The attenuations corresponding to $a^{\prime}$ and $\tilde{a}$ will be denoted by $E^{\prime}$ and $\tilde{E}$, respectively.

The boundary value problem (11) and (3) is equivalent to the operator equation

$$
(I-M) u=J f_{-},
$$

where, using (76),

$$
\begin{aligned}
J f_{-}(x, \theta) & =E\left(x-\tau_{-}(x, \theta) \theta, x\right) f_{-}\left(x-\tau_{-}(x, \theta) \theta, \theta\right) \\
K f(x, \theta) & =\int_{S^{1}} k\left(x, \theta^{\prime}, \theta\right) f\left(x, \theta^{\prime}\right) d \theta^{\prime}, \quad \text { and } \\
M f(x, \theta) & =\int_{0}^{\infty} E(x-t \theta, x) K f(x-t \theta, \theta) d t
\end{aligned}
$$

Under the subcritical assumption

$$
R\|k\|_{\infty}<1 / 2
$$


the operator $M: L^{\infty}\left(B_{R} \times S^{1}\right) \rightarrow L^{\infty}\left(B_{R} \times S^{1}\right)$ is contractive and (177) has a unique solution obtained by Neumann series. Moreover, for $f_{-} \in L^{\infty}\left(\Gamma_{-}^{R}\right)$, we get $u=(I-M)^{-1} J f_{-} \in D(T)$ has a well defined trace in $L^{\infty}\left(\Gamma_{+}^{R}\right)$ given by

$$
\gamma[u](x, \theta):=\left.\left[(I-M)^{-1} J f_{-}\right]\right|_{\Gamma_{+}^{R}}(x, \theta),(x, \theta) \in \Gamma_{+}^{R} ;
$$

see [12, 29]. Therefore the albedo operator $\mathcal{A}: L^{\infty}\left(\Gamma_{-}^{R}\right) \rightarrow L^{\infty}\left(\Gamma_{+}^{R}\right)$ is bounded and has the Schwartz kernel $\alpha\left(x, \theta, x^{\prime}, \theta^{\prime}\right)=\left.\phi\right|_{\Gamma_{+}^{R}}\left(x, \theta ; x^{\prime}, \theta^{\prime}\right)$, where, for $\left(x^{\prime}, \theta^{\prime}\right) \in \Gamma_{-}^{R}$, the map $(x, \theta) \mapsto \phi\left(x, \theta ; x^{\prime}, \theta^{\prime}\right)$ is the fundamental solution of (1) subject to the boundary condition

$$
\left.\phi\right|_{\Gamma_{-}^{R}}\left(\cdot, \cdot ; x^{\prime}, \theta^{\prime}\right)=\left|n\left(x^{\prime}\right) \cdot \theta^{\prime}\right|^{-1} \delta_{\left\{x^{\prime}\right\}}(\cdot) \delta_{\left\{\theta^{\prime}\right\}}(\cdot) .
$$

More precisely, as shown in [29, Proposition 1], $\alpha=\alpha_{0}+\alpha_{1}+\alpha_{2}$ with

$$
\alpha_{0}+\alpha_{1}+\alpha_{2}=\gamma \phi_{0}+\gamma \phi_{1}+\gamma \phi_{2}:=\left.\phi_{0}\right|_{\Gamma_{+}^{R}}+\left.M \phi_{0}\right|_{\Gamma_{+}^{R}}+\left.(I-M)^{-1} M^{2} \phi_{0}\right|_{\Gamma_{+}^{R}},
$$

where

$$
\begin{aligned}
\phi_{0} & =E\left(x-\tau_{-}(x, \theta) \theta, x\right) \delta_{\left\{\theta^{\prime}\right\}}(\theta) \int_{0}^{\tau_{+}\left(x^{\prime}, \theta^{\prime}\right)} \delta\left(x-x^{\prime}-t \theta^{\prime}\right) d t, \\
\phi_{1} & =\frac{\chi(y) k\left(y, \theta^{\prime}, \theta\right)}{\left|\theta^{\prime} \times \theta\right|} E\left(y-\tau_{-}\left(y, \theta^{\prime}\right) \theta^{\prime}, y\right) E\left(y, y+\tau_{+}(y, \theta) \theta\right), \\
0 \leq \phi_{2} & \leq d_{R}\|k\|_{\infty}^{2}\left(1-\ln \left|\theta^{\prime} \times \theta\right|\right) .
\end{aligned}
$$

The constant $d_{R}$ depends on $R$ only, $\chi$ is the characteristic function of $B_{R}$, and, for $\left(x, \theta, x^{\prime}, \theta^{\prime}\right) \in B_{R} \times S^{1} \times \Gamma_{-}^{R}, y=y\left(x, \theta, x^{\prime}, \theta^{\prime}\right)$ is the point of intersection of the rays $x^{\prime} \rightarrow x^{\prime}+\infty \theta^{\prime}$ and $x \rightarrow x-\infty \theta$. The kernel $\beta$ in (16) is then given by

$$
\beta\left(x, \theta, x^{\prime}, \theta^{\prime}\right)=\left[\gamma \phi_{1}+\gamma \phi_{2}\right]\left(x, \theta, x^{\prime}, \theta^{\prime}\right),\left(x, \theta, x^{\prime}, \theta^{\prime}\right) \in \Gamma_{+}^{R} \times \Gamma_{-}^{R},
$$

where $\gamma$ is the trace operator on $\Gamma_{+}^{R}$.

The following estimate from [29] is needed later.

Lemma 6.1. Let $\chi$ be the characteristic function of $B_{R}, L\left(x^{\prime}, \theta^{\prime}\right)$ be the line through $x^{\prime}$ in the direction $\theta^{\prime}$ and $d l(y)$ be the Lebesgue measure on the line. Then

$$
\int_{0}^{\infty} \chi(x-t \theta) \int_{L\left(x^{\prime}, \theta^{\prime}\right)} \frac{\chi(y)}{|x-t \theta-y|} d l(y) d t \leq C\left(1-\ln \left|\theta^{\prime} \times \theta\right|\right),
$$

where $C$ is a constant dependent on $R$ only.

\section{Stability of the Equivalence Classes in TWO Dimensions}

In this section we work under the hypotheses in Section 6 and prove Theorem 1.3 ,

Let $(a, k),(\tilde{a}, \tilde{k}) \in V_{\Sigma, \rho}$ be given with $\|\mathcal{A}-\tilde{\mathcal{A}}\|_{*}=\varepsilon$. Define the pair $\left(a^{\prime}, k^{\prime}\right)$ in the equivalence class of $\langle a, k\rangle$ by (65) and (66) as before, i.e.

$$
a^{\prime}(x, \theta):=a(x, \theta)-\theta \cdot \nabla_{x} \ln \tilde{\varphi}(x, \theta), \quad k^{\prime}\left(x, \theta^{\prime}, \theta\right):=\frac{\tilde{\varphi}(x, \theta)}{\tilde{\varphi}\left(x^{\prime}, \theta^{\prime}\right)} k\left(x, \theta^{\prime}, \theta\right),
$$

where $\tilde{\varphi}$ is given in $\underline{64}$. 
Then the corresponding albedo operator $\mathcal{A}^{\prime}=\mathcal{A}$ and, thus,

$$
\left\|\mathcal{A}^{\prime}-\tilde{\mathcal{A}}\right\|_{*}=\|\mathcal{A}-\tilde{\mathcal{A}}\|_{*}=\varepsilon .
$$

In particular,

$$
\left\|\tilde{\beta}-\beta^{\prime}\right\|_{\infty} \leq \varepsilon
$$

and (60) holds.

Starting with (60), the same arguments as the ones in the three dimensional domains, notably (68), are valid in two dimensions to conclude the estimate (69):

$$
\left\|\tilde{a}-a^{\prime}\right\|_{\infty} \leq \varepsilon\left(e^{2 R \Sigma} / c_{R}\right)=: \varepsilon \tilde{C} .
$$

In turn, (91) yields

$$
\frac{\tilde{\varphi}(x, \theta)}{\tilde{\varphi}\left(x^{\prime}, \theta^{\prime}\right)}=e^{-\int_{0}^{\tau_{-}(x, \theta)}\left(a^{\prime}-\tilde{a}\right)(x-s \theta, \theta) d s+\int_{0}^{\tau_{-}\left(x^{\prime}, \theta^{\prime}\right)}\left(a^{\prime}-\tilde{a}\right)\left(x-s \theta^{\prime}, \theta^{\prime}\right) d s} \leq e^{4 R \tilde{C} \varepsilon} .
$$

From the definition (66) and (92) we now get

$$
\left\|k^{\prime}\right\|_{\infty} \leq \rho e^{4 R \tilde{C} \varepsilon}
$$

Let

$$
\tilde{E}_{1}\left(y, \theta^{\prime}, \theta\right):=\tilde{E}\left(y-\tau_{-}\left(y, \theta^{\prime}\right) \theta^{\prime}\right) \tilde{E}\left(y, y+\tau_{+}(y, \theta) \theta\right) .
$$

be the total attenuation along the broken path due to one scattering at $y \in B_{R}$, when coming from the direction $\theta^{\prime}$ and scattering into the direction $\theta$. The formula (85) now reads

$$
\tilde{\phi}_{1}\left(x, \theta, x^{\prime}, \theta^{\prime}\right)=\frac{\chi(y)\left[\tilde{k} \tilde{E}_{1}\right]\left(y, \theta^{\prime}, \theta\right)}{\left|\theta^{\prime} \times \theta\right|},\left(x, \theta, x^{\prime}, \theta^{\prime}\right) \in B_{R} \times S^{1} \times \Gamma_{-},
$$

where $\chi(y)$ and $y=y\left(x, \theta, x^{\prime}, \theta^{\prime}\right)$ are as described above in Section6. We also consider $E_{1}^{\prime}$ and $\phi_{1}^{\prime}$ defined similarly with the attenuation $a^{\prime}$ to replace $\tilde{a}$.

The relation with the quantity in (43), $F^{\prime}\left(t, x^{\prime}, \theta^{\prime}, \theta\right)=E_{1}^{\prime}\left(x^{\prime}+t \theta^{\prime}, \theta^{\prime}, \theta\right)$, allows us to use the estimates (70) and (771) to conclude

$$
\begin{aligned}
& \left|E_{1}^{\prime}\left(y, \theta^{\prime}, \theta\right)\right| \geq e^{-4 R \Sigma},\left(y, \theta^{\prime}, \theta\right) \in B_{R} \times S^{1} \times S^{1}, \\
& \left\|\tilde{E}_{1}-E_{1}^{\prime}\right\|_{\infty} \leq 2 \varepsilon e^{2 R \Sigma}, .
\end{aligned}
$$

Now use (96), (97), (95) and (87) in $E_{1}^{\prime}\left(\tilde{k}-k^{\prime}\right)=\left(E_{1}^{\prime}-\tilde{E}_{1}\right) \tilde{k}+\left(\tilde{E}_{1} \tilde{k}-E_{1}^{\prime} k^{\prime}\right)$, (evaluated at $\left(y, \theta^{\prime}, \theta\right)$ with $y=y\left(x, \theta, x^{\prime}, \theta^{\prime}\right)$ as above, ) to estimate

$$
\begin{aligned}
e^{-4 R \Sigma}\left|\tilde{k}-k^{\prime}\right| & \leq\left|E_{1}^{\prime}-\tilde{E}_{1}\right| \tilde{k}+\left|\tilde{E}_{1} \tilde{k}-E_{1}^{\prime} k^{\prime}\right| \\
& \leq\left|E_{1}^{\prime}-\tilde{E}_{1}\right| \tilde{k}+\left|\tilde{\beta}-\beta^{\prime}\right|\left|\theta \times \theta^{\prime}\right|+\left|\gamma \phi_{2}^{\prime}-\gamma \tilde{\phi}_{2}\right|\left|\theta \times \theta^{\prime}\right| \\
& \leq 2 \varepsilon \rho e^{2 R \Sigma}+\varepsilon+\left|\gamma \phi_{2}^{\prime}-\gamma \tilde{\phi}_{2}\right|\left|\theta \times \theta^{\prime}\right| .
\end{aligned}
$$


We estimate the last term using the identity

$$
\begin{aligned}
\left|\theta^{\prime} \times \theta\right|\left|\gamma \phi_{2}^{\prime}-\gamma \tilde{\phi}_{2}\right| & =\left|\theta^{\prime} \times \theta\right| \gamma\left(I-M^{\prime}\right)^{-1} M^{\prime 2} \phi_{0}^{\prime}-\left|\theta^{\prime} \times \theta\right| \gamma(I-\tilde{M})^{-1} \tilde{M}^{2} \tilde{\phi}_{0} \\
& =\left|\theta^{\prime} \times \theta\right| \gamma\left(I-M^{\prime}\right)^{-1}\left[M^{\prime 2} \phi_{0}^{\prime}-\tilde{M}^{2} \tilde{\phi}_{0}^{2}\right] \\
& +\left|\theta^{\prime} \times \theta\right| \gamma(I-\tilde{M})^{-1}\left[M^{\prime}-\tilde{M}\right]\left(I-M^{\prime}\right)^{-1} \tilde{M}^{2} \tilde{\phi}_{0}
\end{aligned}
$$

To estimate the first term in the right hand side above we write

$$
\left[M^{\prime 2} \phi_{0}^{\prime}-\tilde{M}^{2} \tilde{\phi}_{0}^{2}\right]=M^{\prime}\left(M^{\prime}-\tilde{M}\right) \phi_{0}^{\prime}+\left(M^{\prime}-\tilde{M}\right) \tilde{M} \phi_{0}^{\prime}+\tilde{M}^{2}\left(\phi_{0}^{\prime}-\tilde{\phi}_{0}\right)
$$

and bound each of the terms as follows. From their definitions we have

$$
\begin{aligned}
\tilde{M} M^{\prime} \phi_{0}^{\prime} & =\int_{0}^{\infty} \tilde{E}(x-t \theta, x) d t \times \\
& \times \int_{L\left(x^{\prime}, \theta^{\prime}\right)} \frac{\tilde{k}(x-t \theta, x \widehat{-t \theta}-y, \theta) k^{\prime}\left(y, \theta^{\prime}, x \widehat{-t \theta-y}\right)}{|x-t \theta-y|} E^{\prime}\left(x^{\prime}, y\right) E^{\prime}(y, x-t \theta) d l(y) . \\
M^{\prime} M^{\prime} \phi_{0}^{\prime} & =\int_{0}^{\infty} E^{\prime}(x-t \theta, x) d t \times \\
& \times \int_{L\left(x^{\prime}, \theta^{\prime}\right)} \frac{k^{\prime}(x-t \theta, x \widehat{-t \theta-y}, \theta) k^{\prime}\left(y, \theta^{\prime}, x \widehat{-t \theta-y)}\right.}{|x-t \theta-y|} E^{\prime}\left(x^{\prime}, y\right) E^{\prime}(y, x-t \theta) d l(y) .
\end{aligned}
$$

Since $\left(I-M^{\prime}\right)^{-1}$ is bounded in $L^{\infty}$, with a norm dependent on the radius only, say $C(R)$, by adding and subtracting one term and by using Lemma 6.1, we estimate

$$
\begin{aligned}
\left|\gamma\left(I-M^{\prime}\right)^{-1}\left(\tilde{M}-M^{\prime}\right) M^{\prime} \phi_{0}^{\prime}\right| \leq & C(R)\left\|\tilde{E}-E^{\prime}\right\|_{\infty}\|k\|_{\infty}\left\|k^{\prime}\right\|_{\infty}\left(1-\ln \mid \theta^{\prime} \times \theta\right) \\
& +C(R)\left\|\tilde{k}-k^{\prime}\right\|_{\infty}\left\|k^{\prime}\right\|_{\infty}\left(1-\ln \left|\theta^{\prime} \times \theta\right|\right) .
\end{aligned}
$$

Now, from (91) we get

$$
\left\|\tilde{E}-E^{\prime}\right\|_{\infty} \leq 2 R\left\|\tilde{a}-a^{\prime}\right\| \leq C\left(R, \Sigma, c_{R}\right) \varepsilon,
$$

for some constant which only depends on $R, \Sigma, c_{R}$.

In what follows we keep the notation $C\left(R, \Sigma, c_{R}\right)$ for constants that may be different from equation to equation but they only depend on $R, \Sigma, c_{R}$ in an explicit, but inessential, way.

Using the fact that $0 \leq t(1-\ln t) \leq 1$ for $t \in[0,1]$, the bound $\|\tilde{k}\| \leq \rho$ and the bound in (93), we get from (100) that

$$
\left|\theta^{\prime} \times \theta \| \gamma\left(I-M^{\prime}\right)^{-1}\left(\tilde{M}-M^{\prime}\right) M^{\prime} \phi_{0}^{\prime}\right| \leq C\left(R, \Sigma, c_{R}\right)\left(\varepsilon \rho^{2}+\rho\left\|\tilde{k}-k^{\prime}\right\|_{\infty}\right) .
$$

By reversing the roles of $M^{\prime}$ and $\tilde{M}$, we get similarly

$$
\left|\theta^{\prime} \times \theta \| \gamma\left(I-M^{\prime}\right)^{-1}\left(M^{\prime}-\tilde{M}\right) \tilde{M} \phi_{0}^{\prime}\right| \leq C\left(R, \Sigma, c_{R}\right)\left(\varepsilon \rho^{2}+\rho\left\|\tilde{k}-k^{\prime}\right\|_{\infty}\right) .
$$

Similarly, from the definition of $\tilde{M}^{2}$ as above, we also get

$$
\left|\theta^{\prime} \times \theta\right| \mid \gamma\left(I-M^{\prime}\right)^{-1} \tilde{M}^{2}\left(\phi_{0}^{\prime}-\tilde{\phi}_{0}\right) \leq C\left(R, \Sigma, c_{R}\right) \varepsilon \rho^{2} .
$$


The estimates (102), (103) and (104) imply

$$
\left|\theta^{\prime} \times \theta\right| \gamma\left(I-M^{\prime}\right)^{-1}\left[M^{\prime 2} \phi_{0}^{\prime}-\tilde{M}^{2} \tilde{\phi}_{0}^{2}\right] \leq C\left(R, \Sigma, c_{R}\right)\left(\varepsilon \rho^{2}+\rho\left\|\tilde{k}-k^{\prime}\right\|_{\infty}\right) .
$$

Next we estimate the second term of the right hand side of (99). Since for any $f \in L^{\infty}\left(B_{R} \times S^{1}\right)$, we have

$$
\begin{aligned}
\left|\left[M^{\prime}-\tilde{M}\right] f(x, \theta)\right| & \leq\left|\int_{0}^{\infty}\left[E^{\prime}-\tilde{E}\right](x-t \theta, x) \int_{S^{1}} k^{\prime}\left(x-t \theta, \theta^{\prime}, \theta\right) f(x-t \theta) d \theta^{\prime} d t\right| \\
& +\left|\int_{0}^{\infty} \tilde{E}(x-t \theta, x) \int_{S^{1}}\left[k^{\prime}-\tilde{k}\right]\left(x-t \theta, \theta^{\prime}, \theta\right) f(x-t \theta) d \theta^{\prime} d t\right| \\
& \leq\left\{2 R\left\|E^{\prime}-\tilde{E}\right\|_{\infty}\left\|k^{\prime}\right\|_{\infty}+2 R\left\|k^{\prime}-\tilde{k}\right\|_{\infty}\right\}\|f\|_{\infty} \\
& \leq\left\{C\left(R, \Sigma, c_{R}\right) \varepsilon \rho+2 R\left\|k^{\prime}-\tilde{k}\right\|_{\infty}\right\}\|f\|_{\infty}
\end{aligned}
$$

we get

$$
\left|\theta^{\prime} \times \theta\right|\left|\gamma(I-\tilde{M})^{-1}\left(M^{\prime}-\tilde{M}\right)^{-1} \tilde{M}^{2} \tilde{\phi}_{0}\right| \leq C\left(R, \Sigma, c_{R}\right)\left\{\varepsilon \rho^{3}+\left\|k^{\prime}-\tilde{k}\right\|_{\infty} \rho^{2}\right\} .
$$

Since $\rho \leq 1$, by applying (105) and (106) in (99), we get

$$
\left|\gamma\left[\phi_{2}^{\prime}\right]-\gamma\left[\tilde{\phi}_{2}\right]\right|\left|\theta \times \theta^{\prime}\right| \leq C\left(R, \Sigma, c_{R}\right)\left\{\varepsilon \rho^{2}+\rho\left\|\tilde{k}-k^{\prime}\right\|_{\infty}\right\} .
$$

Therefore the basic estimate (98) yields

$$
\left\|k^{\prime}-\tilde{k}\right\|_{\infty} \leq C\left(R, \Sigma, c_{R}\right) \varepsilon+C\left(R, \Sigma, c_{R}\right) \rho\left\|k^{\prime}-\tilde{k}\right\|_{\infty} .
$$

By choosing

$$
\rho<\frac{1}{C\left(R, \Sigma, c_{R}\right)}
$$

we get the final estimate

$$
\left\|k^{\prime}-\tilde{k}\right\|_{\infty} \leq \frac{C\left(R, \Sigma, c_{R}\right)}{1-C\left(R, \Sigma, c_{R}\right)} \varepsilon .
$$

The constant $C$ from Theorem 1.3 is the largest between the constant in (91) and (110).

\section{Concluding Remarks}

In the case of an anisotropic attenuating medium, the albedo operator determines the attenuation and scattering properties up to a gauge equivalence class. The set of gauge functions has a natural structure of a multiplicative group which acts transitively on the pairs of the coefficients.

We showed that the gauge equivalent classes are stably determined by the albedo operator. We understand the distance between equivalent classes to be the infimum of the distances between the corresponding representatives.

The proof uses essentially the fact that, without loss of generality, the problem can be transferred to a larger domain, and, consequently, the total travel time (with respect to the larger domain) of free moving particles in the interior domain stays 
away from zero. The no loss of generality part is due to the extension of an estimate in [7] to essentially bounded coefficients.

The fact that we get Lipschitz stability estimates in (13), (14) instead of conditional Hölder stability estimates as in [7] may seem strange. In fact, if we assume that $a$ and $\tilde{a}$ depend on $x$ only (or on $(x,|\theta|)$, if $\theta$ belongs to an open velocity space), then (13) implies

$$
\int(a-\tilde{a})(x+t \theta) d t=O(\varepsilon)
$$

in the $L^{\infty}$ norm, compare with [7, Theorem 3.2]. Then, by using interpolation estimates, and the stability of the X-ray transform, we can get a conditional Hölder stability estimate for $a-\tilde{a}$, similar to the one in [7, Theorem 3.4].

\section{ACKNOWLEDGMENT}

The third author thanks Jason Swanson for pointing out the approximation of the identity result in [14, Theorem 8.15].

\section{REFERENCES}

[1] D. S. Anikonov, The unique determination of the coefficients and the right side in a transport equation, Differential Equations 11(1975), pp. 6-12.

[2] Multidimensional inverse problem for the transport equation, Differential Equations 20(1984), pp. 608-614.

[3] Yu. Anikonov and B. A. Bubnov, Inverse problem of transport theory, Soviet Math. Dokl. 37(1988), pp. 497-499.

[4] H. Babovsky, Identification of Scattering Media from Reflected Flows, SIAM J. Appl. Math. 51(1991), pp. 1674-1704.

[5] G. BAL, Inverse problems for homogeneous transport equations. II. The multidimensional case., Inverse Problems 16(2000), pp. 1013-1028.

[6] - Inverse Transport theory and applications, Inverse Problems (2009), to appear .

[7] G. Bal And A. Jollivet, Stability estimates in stationary inverse transport, Inverse Probl. Imaging 2(2008), pp. 427-454.

[8] G. Bal, I. LAngmore And F. Monard, Inverse transport with isotropic sources and angularly averaged measurements, Inverse Probl. Imaging 2(2008), pp. 23-42.

[9] A. Bondarenko, The structure of the fundamental solution of the time-independent transport equation, J. Math. Anal. Appl. 221(1998), no. 2, 430-451.

[10] K. M. Case And P. F. Zweifel, Linear Transport Theory, Addison-Wesley series in nuclear engineering, Addison-Wesley, Reading, Mass., 1967.

[11] M. Choulli And P. Stefanov, Inverse scattering and inverse boundary value problems for the linear Boltzmann equation, Comm. P.D.E. 21(1996), 763-785.

[12] An inverse boundary value problem for the stationary transport, Osaka J. Math. 36(1999), pp. 87-104.

[13] R. Dautray And J.-L. Lions, Mathematical Analysis and Numerical Methods for Science and Technology. Vol.6, Springer Verlag, Berlin, 1993.

[14] G. Folland, Real Analysis, Modern Techniques and Their Applications, John Wiley \&Sons, New York, 1984.

[15] Y. Kurylev, M. Lassas, G. Uhlmann, Rigidity of broken geodesic flow and inverse problems, to appear in American Journal of Mathematics.

[16] I. LANGMORE, The stationary transport problem with angularly averaged measurements, Inverse Problems 24(2008), no. 1, 015024. 
[17] I. Langmore And S. McDowall, Optical tomography for variable refractive index with angularly averaged measurements, Comm. PDE, 33 (2008), 2180-2207.

[18] E. W. Larsen, Solution of multidimensional inverse transport problems, J. Math. Phys. 25(1984), 131-135.

[19] Solution of the three dimensional inverse transport problems, Transport Theory and Stat. Phys. 17(2\&3)(1988), 147-167.

[20] N. J. McConmick, Inverse radiative transfer problems: a review, Nucl. Sci. Eng. 112(1992), pp. $185-198$.

[21] S. MCDowall, Inverse problem for the transport equation in the presence of a Riemannian metric, Pac. J. Math., 216 (2004), no.1, 107-129.

[22] _ Optical Tomography on Simple Riemannian Surfaces, Comm. PDE 30(2005), pp. 1379 -1400 .

[23] S. McDowall, P. Stefanov and A. Tamasan, Gauge equivalence in stationary radiative transport through media with a variable index of refraction, submitted 2009.

[24] M. Mokhtar-Kharroubi, Mathematical Topics in Neutron Transport Theory, World Scientific, Singapore, 1997.

[25] M. Reed And B. Simon, Methods of Modern Mathematical Physics, Vol. 3, Academic Press, New York, 1979.

[26] M. Rivaric And I. Vidav, Analytic properties of the inverse $A(z)^{-1}$ of an analytic linear operator valued function A(z), Arch. Rat. Mech. Anal. 32(4)(1969), 298-310.

[27] V. Romanov, Stability estimates in problems of recovering the attenuation coefficient and the scattering indicatrix for the transport equation, J. Inverse Ill-Posed Probl. 4(1966)(4), 297-305.

[28] P. Stefanov and A. Tamasan Uniqueness and non-uniqueness in inverse radiative transfer, Proc. Amer. Math. Soc. 137(2009), pp. 2335-2344.

[29] P. Stefanov and G. Uhlmann, Optical tomography in two dimensions, Methods Appl. Anal. 10(2003), pp. 1-9.

[30] _ An inverse source problem in optical molecular imaging, Analysis and PDE 1(2008), pp. $115-126$.

[31] A. Tamasan, An inverse boundary value problem in two-dimensional transport, Inverse Problems 18(2002), pp. 209-219.

[32] _ Optical tomography in weakly anisotropic scattering media, Contemporary Mathematics 333(2003), pp. 199-207.

[33] J.-N. Wang, Stability estimates of an inverse problem for the stationary transport, Ann. Inst. Henri Poincaré 70(1999), pp. 473-495.

Stephen McDowall, Department of Mathematics, Western Washington University, 516 High Street, Bellingham, WA 98225-9063

E-mail address: stephen.mcdowall@wwu.edu

Plamen Stefanov, Department of Mathematics, Purdue University, 150 N. University Street, West Lafayette, IN 47907-2067

E-mail address: stefanov@math.purdue.edu

Alexandru Tamasan, Department of Mathematics, University of Central Florida, 4000 Central Florida Blvd., Orlando, FL, 32816, USA

E-mail address: tamasan@math.ucf.edu 